\title{
Early Endothelial Dysfunction in Type 1 Diabetes Is Accompanied by an Impairment of Vascular Smooth Muscle Function: A Meta-Analysis
}

\section{OPEN ACCESS}

Edited by:

Gaetano Santulli,

Columbia University, United States

Reviewed by:

Yoshifumi Saisho,

Keio University School of

Medicine, Japan

Philippz Obert,

University of Avignon, France

*Correspondence:

Elsa Heyman

elsa.heyman@univ-lille.fr

tThese authors have contributed equally to this work

Specialty section: This article was submitted to

Clinical Diabetes,

a section of the journal

Frontiers in Endocrinology

Received: 10 January 2020

Accepted: 23 March 2020

Published: 17 April 2020

Citation:

Lespagnol E, Dauchet L,

Pawlak-Chaouch M, Balestra C,

Berthoin S, Feelisch M, Roustit M, Boissière J, Fontaine $P$ and Heyman $E$ (2020) Early Endothelial Dysfunction in

Type 1 Diabetes Is Accompanied by an Impairment of Vascular Smooth Muscle Function: A Meta-Analysis.

Front. Endocrinol. 11:203.

doi: 10.3389/fendo.2020.00203
Elodie Lespagnol ${ }^{1}$, Luc Dauchet ${ }^{2 \dagger}$, Mehdi Pawlak-Chaouch ${ }^{1 \dagger}$, Costantino Balestra ${ }^{3}$, Serge Berthoin ${ }^{1}$, Martin Feelisch ${ }^{4}$, Matthieu Roustit ${ }^{5}$, Julien Boissière ${ }^{1}$, Pierre Fontaine ${ }^{6}$ and Elsa Heyman ${ }^{1 *}$

${ }^{1}$ Univ. Lille, Univ. Artois, Univ. Littoral Côte d'Opale, ULR 7369 - URePSSS - Unité de Recherche Pluridisciplinaire Sport Santé Société, Lille, France, ${ }^{2}$ Univ. Lille, Inserm, CHU Lille, Institut Pasteur de Lille, U1167 - RID-AGE - Facteurs de risque et déterminants moléculaires des maladies liées au vieillissement, Lille, France, ${ }^{3}$ Environmental and Occupational (Integrative) Physiology Laboratory, Haute École Bruxelles-Brabant HE2B, Brussels, Belgium, ${ }^{4}$ Clinical and Experimental Sciences, Faculty of Medicine, University Hospital Southampton NHS Foundation Trust, University of Southampton, Southampton, United Kingdom, ${ }^{5}$ Univ. Grenoble Alpes, HP2, Inserm, CHU Grenoble Alpes, Grenoble, France, ${ }^{6}$ Département d'endocrinologie, Diabète et maladies métaboliques, Hôpital Huriez, Université de Lille, Lille, France

Background: A large yet heterogeneous body of literature exists suggesting that endothelial dysfunction appears early in type 1 diabetes, due to hyperglycemia-induced oxidative stress. The latter may also affect vascular smooth muscles (VSM) function, a layer albeit less frequently considered in that pathology. This meta-analysis aims at evaluating the extent, and the contributing risk factors, of early endothelial dysfunction, and of the possible concomitant VSM dysfunction, in type 1 diabetes.

Methods: PubMed, Web of Sciences, Cochrane Library databases were screened from their respective inceptions until October 2019. We included studies comparing vasodilatory capacity depending or not on endothelium (i.e., endothelial function or VSM function, respectively) in patients with uncomplicated type 1 diabetes and healthy controls.

Results: Fifty-eight articles studying endothelium-dependent function, among which 21 studies also assessed VSM, were included. Global analyses revealed an impairment of standardized mean difference (SMD) (Cohen's d) of endothelial function: -0.61 (95\% Cl: $-0.79,-0.44)$ but also of VSM SMD: -0.32 (95\% Cl: $-0.57,-0.07)$. The type of stimuli used (i.e., exercise, occlusion-reperfusion, pharmacological substances, heat) did not influence the impairment of the vasodilatory capacity. Endothelial dysfunction appeared more pronounced within macrovascular than microvascular beds. The latter was particularly altered in cases of poor glycemic control $\left[\mathrm{HbA}_{1 \mathrm{c}}>67 \mathrm{mmol} / \mathrm{mol}(8.3 \%)\right]$.

Conclusions: This meta-analysis not only corroborates the presence of an early impairment of endothelial function, even in response to physiological stimuli like exercise, but also highlights a VSM dysfunction in children and adults with type 1 diabetes. Endothelial dysfunction seems to be more pronounced in large than small vessels, fostering the debate on their relative temporal appearance.

Keywords: endothelial function, exercise, macrocirculation, microcirculation, peripheral vascular disease, smooth muscle function, type 1 diabetes 


\section{INTRODUCTION}

Despite significant advances in diabetes care, individuals with type 1 diabetes remain to be prone to the development of comorbidities, in particular those associated with vascular complications. Type 1 diabetes is associated with a 2 - to 10 -fold higher mortality and cardiovascular disease risk (1). Structural and functional endothelial aberrations seem to occur in small and large blood vessels early during diabetes development, long before the manifestation of overt micro- or macro-vascular complications (2-4). The associated endothelial dysfunction is now accepted as a reliable predictor of cardiovascular disease $(5,6)$. Alarming data suggest a $>35 \%$ prevalence of endothelial dysfunction in individuals within 5 years of type 1 diabetes (7). Endothelial dysfunction in diabetes may be the result of a combination of multiple stressors including hyperglycaemia and oxidative stress $(1,8)$. Acting in concert, these factors lead to a decrease in the bioavailability of nitric oxide (NO). Vascular homeostasis depends to a significant extent on the capacity of the endothelium to produce NO. While one of the principal functions that led to the discovery of NO in the cardiovascular system is to relax vascular smooth muscle by enhancing cyclic guanosine monophosphate (cGMP) production, this is only part of the story. $\mathrm{NO}$ is also a potent antioxidant and a regulator of local and systemic redox status (4), and these facets may play a more significant role in metabolic disease settings than hitherto assumed.

Much has been written on the subject of early endothelial dysfunction in type 1 diabetes. However, this large body of literature remains complex, with numerous contradictory findings (Table 1). It should be emphasized that the conventional assessments of endothelial function always include the responsiveness of the vascular smooth muscle (VSM) layer in addition. To better dissect the relative contribution of these two contributors to vascular dysfunction, it is crucial to specifically assess vascular smooth muscle reactivity in parallel to the endothelial function test. However, VSM function is considered in only less than a half of the papers dealing with endothelial function in type 1 diabetes, with contradictory results (Table 1). In vitro evidence strongly suggests a deleterious impact of chronic hyperglycemia on VSM, by provoking a dysregulation of $\mathrm{Ca}^{2+}$ signaling (65) and vascular remodeling (66).

Abbreviations: ach, Acetylcholine; ADMA, Asymmetrical dimethylarginine; BMI, Body Mass Index; cGMP, Cyclic guanosine monophosphate; CI, Confidence Interval; DBP, Diastolic Blood Pressure; EDHF, Endothelial Derived Hyperpolarizing Factor; EET, Epoxyeicosatrienoic acids; eNOS, Endothelial Nitric Oxide Synthase; FMD, Flow-mediated dilation; HbAlc, Glycated Hemoglobin; HC, Healthy Controls; HDL-C, High Density Lipids-Cholesterol; IDDM, InsulinDependent Diabetes Mellitus; L-NMMA, NG-Monomethyl-L-arginine; MACRO, Macrocirculation; MCh, Metacholine; MICRO, Microcirculation; NIRS, NearInfrared Spectroscopy; NMD, Nitrate-Mediated Dilation; NO, Nitric Oxide; NOx, Sum of nitrite and nitrate; PEAK, Data displayed as peak values in the original paper; PORH, Post-occlusive reactive hyperaemia; PP, Post-Prandial; SBP, Systolic Blood Pressure; SD, Standard Deviation; SE, Standard Error; sGC, Guanylate cyclase; SMD, Standard Mean Deviation; SNP, Sodium Nitropusside; TG, Tryglycerides; VAR, Data displayed as variations from baseline in the original paper; VO2, Aerobic capacity; VSM, Vascular smooth muscle.
Apparent inconsistencies about VSM and endothelial dysfunction in the literature may be related to the heterogeneity in conditions (e.g., age, glycemic control, and presence of risk factors) and vascular beds (artery, subcutaneous or muscular capillaries, and arterioles) studied as well as the different measurement methods used [ultrasonography, plethysmography, near-infrared spectroscopy (NIRS), tonometry, laser Doppler, capillaroscopy] and test stimuli applied (pharmacological substances, post-occlusive reactive hyperemia, heat). Although increased metabolic demand following physical exercise is one of the strongest physiological signals for upstream vasodilation, this natural stimulus is rarely used.

Two previous meta-analyses undertook to assess endothelial dysfunction in patients with type 1 diabetes but only in response to one type of stimulus, i.e., the response to occlusionreperfusion at the macrovascular level [FMD (Flow mediated dilation): post-occlusive hyperemia of the brachial artery] (67) or dermal microvascular response to local thermal hyperemia (68). The latter stimuli are however only two of the numerous stimuli investigated in literature for vasoreactivity assessment. In addition, these previous meta-analyses did not take into account the presence or absence of overt vascular complications among patients included. However, in a preventive context, it appears worth dealing with patients still free from clinical complications, when one knows the strong predictive nature of endothelial dysfunction for future cardiovascular disease (5). The present meta-analysis of published data on vasodilatory capacities in patients with type 1 diabetes without complications and healthy controls was conducted to (i) evaluate the extent of early endothelial dysfunction, and assess possible concomitant VSM dysfunction, in type 1 diabetes and (ii) to disentangle, through metaregressions (i.e., sensitivity analyses), which of the many factors contributes most to the development and/or manifestation of the resulting vascular dysfunction.

\section{MATERIALS AND METHODS}

This meta-analysis was conducted according to the PRISMA Statement guidelines (69) and registered in Prospero (IDCRD42019116319). We have followed PICOS recommendations as described throughout this section of manuscript.

\section{Data Sources and Searches}

The searches have been undertaken using three different databases: Pubmed, Cochrane Library, and Web of Sciences until October 2019. All of the following terms, alone and in combination, were used: "type 1 diabetes," "IDDM," "macrovascular," "microvascular," "endothelial function," "endothelium," "exercise," "physical activity," "sport*”, "contraction," "hemodynamic*," "acetylcholine," "sodium nitropusside," "flow-mediated dilation," "hyperaemia," "iontophoresis," "blood flow," "blood pressure," "FMD," "NMD," "vasodilation," "vasodilatation," "vascular," "vasoreactivity." We excluded the terms "type 2 diabetes," "mice," "mouse," "rat." Only articles written in English were included. Articles were selected in the first instance by title and abstract. In the second instance, 
TABLE 1 | Main characteristics of studies included in the current meta-analysis.

\begin{tabular}{|c|c|c|c|c|c|c|c|c|c|c|c|c|c|c|}
\hline \multirow{2}{*}{$\begin{array}{l}\text { Authors and } \\
\text { year of } \\
\text { publication }\end{array}$} & \multicolumn{2}{|c|}{$\begin{array}{c}n \\
\text { (\% women) }\end{array}$} & \multicolumn{2}{|c|}{ Age (years) } & \multirow{2}{*}{$\begin{array}{l}\text { Type 1 } \\
\text { diabetes' } \\
\text { duration } \\
\text { (years) }\end{array}$} & \multirow{2}{*}{$\begin{array}{l}\text { Type } 1 \\
\text { Diabetes } \\
\text { HbA1c } \\
(\%)\end{array}$} & \multirow{2}{*}{$\begin{array}{l}\begin{array}{l}\text { Glycaemia } \\
\text { before } \\
\text { test } \\
\text { (mmol.L) } \\
\text { (F/PP) }\end{array} \\
\begin{array}{l}\text { Type } 1 \\
\text { diabetes }\end{array}\end{array}$} & \multicolumn{4}{|c|}{ Quality scores } & \multirow{2}{*}{$\begin{array}{l}\text { Vascular } \\
\text { region } \\
\text { assessed } \\
\text { (VAR/PEAK) }\end{array}$} & \multirow{2}{*}{$\begin{array}{l}\text { Endothelium- } \\
\text { dependent } \\
\text { function (EF) } \\
\text { Time and place } \\
\text { of occlusion or } \\
\text { characteristic of } \\
\text { exercise } \\
\text { (technique) }\end{array}$} & \multirow{2}{*}{$\begin{array}{l}\text { Endotheliur } \\
\text { independer } \\
\text { function } \\
\text { (VSM) }\end{array}$} \\
\hline & $\begin{array}{l}\text { Type } 1 \\
\text { diabetes }\end{array}$ & $\mathrm{HC}$ & $\begin{array}{l}\text { Type } 1 \\
\text { diabetes }\end{array}$ & $\mathrm{HC}$ & & & & Complications & $\begin{array}{c}\text { Age } \\
\text { matching }\end{array}$ & $\begin{array}{l}\text { Gender } \\
\text { matching }\end{array}$ & $\begin{array}{c}\text { BMI } \\
\text { matching }\end{array}$ & & & \\
\hline $\begin{array}{l}\text { Abd El Dayem } \\
\text { et al. (9) }\end{array}$ & $\begin{array}{l}62^{\dagger} \\
(50)\end{array}$ & $\begin{array}{l}30^{\dagger} \\
(50)\end{array}$ & $16.1 \pm 2.6$ & $16.1 \pm 2.6$ & $8.9 \pm 3.11$ & $9.5 \pm 1.9$ & $\begin{array}{l}\text { NA } \\
(F)\end{array}$ & 0 & 2 & 2 & 1 & $\begin{array}{l}\text { MACRO } \\
\text { artery } \\
\text { (VAR) }\end{array}$ & $\begin{array}{l}\searrow \text { FMD } \\
5 \text { min on forearm } \\
\text { (ultrasound) }\end{array}$ & $\leftrightarrow \mathrm{NMD}$ \\
\hline $\begin{array}{l}\text { Abi-Chahin } \\
\text { et al., (10) }\end{array}$ & $\begin{array}{l}30^{*} \\
(70)\end{array}$ & $\begin{array}{l}31 \\
(68)\end{array}$ & $23.7 \pm 4.31$ & $23.4 \pm 5.4$ & $12.9 \pm 6.7$ & NA & $\begin{array}{l}\text { NA } \\
\text { (NA) }\end{array}$ & 1 & 2 & 1 & 1 & $\begin{array}{l}\text { MICRO } \\
\text { cutaneous } \\
\text { (VAR) }\end{array}$ & $\begin{array}{l}\searrow \mathrm{PORH} \\
1 \mathrm{~min} \text { on the fourth } \\
\text { finger } \\
\text { (capillaroscopy) }\end{array}$ & NA \\
\hline $\begin{array}{l}\text { Aburawi et al. } \\
\text { (11) }\end{array}$ & $\begin{array}{l}15 \\
(\mathrm{NA})\end{array}$ & $\begin{array}{l}10 \\
\text { (NA) }\end{array}$ & $14 \pm 4.0$ & $14.0 \pm 3.0$ & $5.0 \pm 3.0$ & $7.3 \pm 2.0$ & $\begin{array}{l}\text { NA } \\
\text { (NA) }\end{array}$ & 0 & 2 & 0 & 0 & $\begin{array}{l}\text { MACRO } \\
\text { artery } \\
\text { (VAR) }\end{array}$ & $\begin{array}{l}\searrow \text { FMD } \\
5 \text { min on forearm } \\
\text { (ultrasound) }\end{array}$ & $\leftrightarrow \mathrm{NMD}$ \\
\hline $\begin{array}{l}\text { Allen et al. } \\
\text { (12) }\end{array}$ & $\begin{array}{l}15^{\dagger} \\
(0)\end{array}$ & $\begin{array}{l}15^{\dagger} \\
(0)\end{array}$ & $29.0 \pm 6.0$ & $26.0 \pm 6.0$ & $13.0 \pm 7.0$ & $8.2 \pm 1.3$ & $\begin{array}{l}11.3 \pm 4.6 \\
(\mathrm{PP})\end{array}$ & 0 & 2 & 1 & 1 & $\begin{array}{l}\text { MICRO } \\
\text { cutaneous } \\
\text { (PEAK) }\end{array}$ & $\begin{array}{l}\leftrightarrow \text { During } \\
\text { intermittent local } \\
\text { exercise (1 } \\
\text { contraction per } 4 \mathrm{~s} \\
\text { at } 25 \% \text { maximal } \\
\text { voluntary capacity, } \\
3 \text { min) + } \\
\text { FMD } \\
\text { (plethysmography) } \\
\searrow \text { FMD } 5 \text { min on } \\
\text { upper arm } \\
\text { (plethysmography) }\end{array}$ & NA \\
\hline $\begin{array}{l}\text { Aslan et al. } \\
\text { (13) }\end{array}$ & $\begin{array}{l}76^{*} \\
(50)\end{array}$ & $\begin{array}{l}36^{*} \\
(45)\end{array}$ & $30.6 \pm 10.3$ & $32.4 \pm 8.5$ & $11.7 \pm 8.1$ & $8.9 \pm 1.57$ & $\begin{array}{l}7.9 \pm 3.1 \\
(\mathrm{NA})\end{array}$ & 1 & 2 & 2 & 2 & $\begin{array}{l}\text { MACRO } \\
\text { artery } \\
\text { (VAR) }\end{array}$ & $\begin{array}{l}\searrow \text { FMD } \\
5 \text { min on forearm } \\
\text { (ultrasound) }\end{array}$ & $\leftrightarrow \mathrm{NMD}$ \\
\hline \multicolumn{15}{|c|}{ Oral contraceptive } \\
\hline $\begin{array}{l}\text { Babar et al. } \\
\text { (14) }\end{array}$ & $\begin{array}{l}21 \\
\left(57^{\ddagger}\right)\end{array}$ & $\begin{array}{l}15 \\
\left(60^{\ddagger}\right)\end{array}$ & $8.3 \pm 1.37$ & $7.6 \pm 1.2$ & $4.3 \pm 4.6$ & $8.0 \pm 0.9$ & $\begin{array}{l}\text { NA } \\
(F)\end{array}$ & 0 & 2 & 0 & 0 & $\begin{array}{l}\text { MACRO } \\
\text { artery } \\
\text { (VAR) }\end{array}$ & $\begin{array}{l}\searrow \text { FMD } \\
5 \text { min on forearm } \\
\text { (ultrasound) }\end{array}$ & NA \\
\hline $\begin{array}{l}\text { Bayir et al. } \\
\text { (15) }\end{array}$ & $\begin{array}{l}50 \\
\left(46^{\ddagger}\right)\end{array}$ & $\begin{array}{l}45 \\
\left(47^{\ddagger}\right)\end{array}$ & $12.1 \pm 2.02$ & $11.5 \pm 1.9$ & $3.7 \pm 1.9$ & $9.2 \pm 2.5$ & $\begin{array}{l}\text { NA } \\
(\mathrm{F})\end{array}$ & 1 & 2 & 2 & 1 & $\begin{array}{l}\text { MACRO } \\
\text { artery } \\
\text { (NAR) }\end{array}$ & $\begin{array}{l}\leftrightarrow \text { FMD } \\
3 \text { min on forearm } \\
\text { (ultrasound) }\end{array}$ & NA \\
\hline
\end{tabular}


TABLE 1 | Continued

\begin{tabular}{|c|c|c|c|c|c|c|c|c|c|c|c|c|c|c|}
\hline \multirow{2}{*}{$\begin{array}{l}\text { Authors and } \\
\text { year of } \\
\text { publication }\end{array}$} & \multicolumn{2}{|c|}{$\begin{array}{c}n \\
\text { (\% women) }\end{array}$} & \multicolumn{2}{|c|}{ Age (years) } & \multirow{2}{*}{$\begin{array}{l}\text { Type } 1 \\
\text { diabetes' } \\
\text { duration } \\
\text { (years) }\end{array}$} & \multirow{2}{*}{$\begin{array}{l}\text { Type 1 } \\
\text { Diabetes } \\
\text { HbA1c } \\
(\%)\end{array}$} & \multirow{2}{*}{$\begin{array}{l}\text { Glycaemia } \\
\text { before } \\
\text { test } \\
\text { (mmol.L) } \\
\text { (F/PP) } \\
\\
\begin{array}{l}\text { Type } 1 \\
\text { diabetes }\end{array}\end{array}$} & \multicolumn{4}{|c|}{ Quality scores } & \multirow{2}{*}{$\begin{array}{l}\text { Vascular } \\
\text { region } \\
\text { assessed } \\
\text { (VAR/PEAK) }\end{array}$} & \multirow{2}{*}{$\begin{array}{l}\text { Endothelium- } \\
\text { dependent } \\
\text { function (EF) } \\
\text { Time and place } \\
\text { of occlusion or } \\
\text { characteristic of } \\
\text { exercise } \\
\text { (technique) }\end{array}$} & \multirow{2}{*}{$\begin{array}{l}\text { Endotheliun } \\
\text { independen } \\
\text { function } \\
\text { (VSM) }\end{array}$} \\
\hline & $\begin{array}{l}\text { Type } 1 \\
\text { diabetes }\end{array}$ & $\mathrm{HC}$ & $\begin{array}{l}\text { Type } 1 \\
\text { diabetes }\end{array}$ & $\mathrm{HC}$ & & & & Complications & $\begin{array}{c}\text { Age } \\
\text { matching }\end{array}$ & $\begin{array}{l}\text { Gender } \\
\text { matching }\end{array}$ & $\begin{array}{c}\text { BMI } \\
\text { matching }\end{array}$ & & & \\
\hline \multirow[t]{2}{*}{$\begin{array}{l}\text { Bellien et al. } \\
\text { (16) }\end{array}$} & $\begin{array}{l}16 \\
(54)\end{array}$ & $\begin{array}{l}24^{*} \\
(50)\end{array}$ & NA & $\begin{array}{l}37.0 \pm \\
14.7\end{array}$ & NA & NA & $\begin{array}{l}\mathrm{NA} \\
\text { (NA) }\end{array}$ & 1 & 0 & 1 & 1 & $\begin{array}{l}\text { MACRO } \\
\text { artery } \\
\text { (VAR) }\end{array}$ & $\begin{array}{l}\text { FMD } \\
10 \text { min on the wrist } \\
\text { (ultrasound) }\end{array}$ & $\leftrightarrow \mathrm{NMD}$ \\
\hline & & & & & & & & & & & & & $\begin{array}{l}\searrow \text { Heat } \\
\text { (ultrasound) }\end{array}$ & NA \\
\hline \multirow[t]{2}{*}{$\begin{array}{l}\text { Boolell and } \\
\text { Tooke (17) }\end{array}$} & $\begin{array}{l}6^{\dagger} \\
(17)\end{array}$ & $\begin{array}{l}9^{\dagger} \\
(22)\end{array}$ & $34.0 \pm 11.0$ & $\begin{array}{l}30.0 \pm \\
11.0\end{array}$ & $4.5 \pm 2.9$ & $7.7 \pm 1.8$ & $\begin{array}{l}7.6 \pm 4.0 \\
(P P)\end{array}$ & 1 & 0 & 0 & 0 & $\begin{array}{l}\text { MICRO } \\
\text { cutaneous } \\
\text { (PEAK) }\end{array}$ & $\begin{array}{l}\leftrightarrow \text { Capsaïcine } \\
\text { (laser Doppler) }\end{array}$ & NA \\
\hline & & & & & & & & & & & & & $\begin{array}{l}\searrow \text { Substance P } \\
\text { (laser Doppler) }\end{array}$ & NA \\
\hline $\begin{array}{l}\text { Bradley et al. } \\
\text { (18) }\end{array}$ & $\begin{array}{l}199^{*} \\
\left(51^{\ddagger}\right)\end{array}$ & $\begin{array}{l}178 \\
\left(53^{\ddagger}\right)\end{array}$ & $14.4 \pm 1.6$ & $14.4 \pm 2.1$ & $7.2 \pm 3.1$ & $8.5 \pm 1.2$ & $\begin{array}{l}\text { NA } \\
(\mathrm{F})\end{array}$ & 1 & 2 & 0 & 0 & $\begin{array}{l}\text { MACRO } \\
\text { artery } \\
\text { (VAR) }\end{array}$ & $\begin{array}{l}\searrow F M D \\
5 \text { min on forearm } \\
\text { (ultrasound) }\end{array}$ & NA \\
\hline $\begin{array}{l}\text { Bruzzi et al. } \\
\text { (19) }\end{array}$ & $\begin{array}{l}39 \\
(51)\end{array}$ & $\begin{array}{l}45 \\
(51)\end{array}$ & $11.2 \pm 3.7$ & $10.2 \pm 3.1$ & $4.0 \pm 2.8$ & $8.0 \pm 0.9$ & $\begin{array}{l}13.6 \pm 5.3 \\
(F)\end{array}$ & 0 & 2 & 0 & 1 & $\begin{array}{l}\text { MACRO } \\
\text { artery } \\
\text { (VAR) }\end{array}$ & $\begin{array}{l}\leftrightarrow \text { FMD } \\
4.5 \text { min on forearm } \\
\text { (ultrasound) }\end{array}$ & NA \\
\hline $\begin{array}{l}\text { Calver et al. } \\
\text { (20) }\end{array}$ & $\begin{array}{l}10^{\dagger} \\
(0)\end{array}$ & $\begin{array}{l}10^{\dagger} \\
(0)\end{array}$ & $26.2 \pm 4.7$ & $24.9 \pm 5.1$ & $3.2 \pm 3.1$ & $6.7 \pm 1.6$ & $\begin{array}{l}\mathrm{NA} \\
\text { (NA) }\end{array}$ & 0 & 2 & 2 & 0 & $\begin{array}{l}\text { MICRO } \\
\text { Cutaneous + } \\
\text { muscle(PEAK) }\end{array}$ & $\begin{array}{l}\stackrel{\leftrightarrow}{A C h} \\
\text { (plethysmography) }\end{array}$ & $\searrow S N P$ \\
\hline $\begin{array}{l}\text { Ceriello et al. } \\
\text { (21) }\end{array}$ & $\begin{array}{l}22 \\
(11)\end{array}$ & $\begin{array}{l}20 \\
(8)\end{array}$ & $23.5 \pm 13.6$ & $\begin{array}{l}23.2 \pm \\
13.9\end{array}$ & NA & $8.1 \pm 1.9$ & $\mathrm{NA}(\mathrm{F})$ & 1 & 1 & 0 & 1 & $\begin{array}{l}\text { MACRO } \\
\text { artery } \\
\text { (VAR) }\end{array}$ & $\begin{array}{l}\searrow \text { FMD } \\
5 \text { min on forearm } \\
\text { (ultrasound) }\end{array}$ & NA \\
\hline $\begin{array}{l}\text { Chiesa et al. } \\
\text { (22) }\end{array}$ & $\begin{array}{l}70 \\
(61)\end{array}$ & $\begin{array}{l}30 \\
(47)\end{array}$ & $14.6 \pm 1.7$ & $13.9 \pm 2.1$ & $8.9 \pm 3.8$ & 8.3 & $\begin{array}{l}\text { NA } \\
\text { (NA) }\end{array}$ & 1 & 2 & 0 & 0 & $\begin{array}{l}\text { MACRO } \\
\text { artery } \\
\text { (VAR) }\end{array}$ & $\begin{array}{l}\leftrightarrow \mathrm{FMD} \\
5 \text { min on forearm } \\
\text { (ultrasound) }\end{array}$ & NA \\
\hline $\begin{array}{l}\text { Ciftel et al. } \\
(23)\end{array}$ & $\begin{array}{l}42 \\
\left(N A^{\ddagger}\right)\end{array}$ & $\begin{array}{l}40 \\
\left(N A^{\ddagger}\right)\end{array}$ & $13.2 \pm 2.6$ & $13.1 \pm 2.8$ & $6.9 \pm 1.8$ & $9.0 \pm 1.4$ & $\begin{array}{l}\text { NA } \\
\text { (NA) }\end{array}$ & 1 & 2 & 0 & 2 & $\begin{array}{l}\text { MACRO } \\
\text { artery } \\
\text { (VAR) }\end{array}$ & $\begin{array}{l}\searrow \text { FMD } \\
3 \text { min on forearm } \\
\text { (ultrasound) }\end{array}$ & NA \\
\hline $\begin{array}{l}\text { DiMeglio et al. } \\
\text { (24) }\end{array}$ & $\begin{array}{l}17 \\
(52)\end{array}$ & $\begin{array}{l}18 \\
(50)\end{array}$ & $10.7 \pm 3.5$ & $20.5 \pm 1.4$ & $21.1 \pm 3.5$ & $9.4 \pm 1.6$ & $\begin{array}{l}\text { NA } \\
(F)\end{array}$ & 0 & 0 & 2 & 0 & $\begin{array}{l}\text { MICRO } \\
\text { cutaneous } \\
\text { (VAR) }\end{array}$ & $\begin{array}{l}\searrow \text { ACh } \\
\text { (laser Doppler) }\end{array}$ & NA \\
\hline $\begin{array}{l}\text { Eltayeb et al. } \\
\text { (25) }\end{array}$ & $\begin{array}{l}30 \\
\left(43^{\ddagger}\right)\end{array}$ & $\begin{array}{l}30 \\
\left(43^{\ddagger}\right)\end{array}$ & $11.1 \pm 3.8$ & $9.8 \pm 3.5$ & $3.9 \pm 0.6$ & $9.7 \pm 2.2$ & $\begin{array}{l}12.8 \\
(F)\end{array}$ & 1 & 2 & 2 & 2 & $\begin{array}{l}\text { MACRO } \\
\text { artery } \\
\text { (VAR) }\end{array}$ & $\begin{array}{l}\searrow \text { FMD } \\
4.5 \text { min on forearm } \\
\text { (ultrasound) }\end{array}$ & NA \\
\hline
\end{tabular}


TABLE 1 | Continued

\begin{tabular}{|c|c|c|c|c|c|c|c|c|c|c|c|c|c|c|}
\hline \multirow{2}{*}{$\begin{array}{l}\text { Authors and } \\
\text { year of } \\
\text { publication }\end{array}$} & \multicolumn{2}{|c|}{$\begin{array}{c}n \\
\text { (\% women) }\end{array}$} & \multicolumn{2}{|c|}{ Age (years) } & \multirow{2}{*}{$\begin{array}{l}\text { Type 1 } \\
\text { diabetes' } \\
\text { duration } \\
\text { (years) }\end{array}$} & \multirow{2}{*}{$\begin{array}{l}\text { Type } 1 \\
\text { Diabetes } \\
\text { HbA1c } \\
(\%)\end{array}$} & \multirow{2}{*}{$\begin{array}{l}\text { Glycaemia } \\
\text { before } \\
\text { test } \\
\text { (mmol.L) } \\
\text { (F/PP) } \\
\\
\begin{array}{l}\text { Type 1 } \\
\text { diabetes }\end{array}\end{array}$} & \multicolumn{4}{|c|}{ Quality scores } & \multirow{2}{*}{$\begin{array}{l}\text { Vascular } \\
\text { region } \\
\text { assessed } \\
\text { (VAR/PEAK) }\end{array}$} & \multirow{2}{*}{$\begin{array}{l}\text { Endothelium- } \\
\text { dependent } \\
\text { function (EF) } \\
\text { Time and place } \\
\text { of occlusion or } \\
\text { characteristic of } \\
\text { exercise } \\
\text { (technique) }\end{array}$} & \multirow{2}{*}{$\begin{array}{c}\text { Endothelium } \\
\text { independen } \\
\text { function } \\
\text { (VSM) }\end{array}$} \\
\hline & $\begin{array}{l}\text { Type } 1 \\
\text { diabetes }\end{array}$ & $\mathrm{HC}$ & $\begin{array}{l}\text { Type } 1 \\
\text { diabetes }\end{array}$ & $\mathrm{HC}$ & & & & Complications & $\begin{array}{c}\text { Age } \\
\text { matching }\end{array}$ & $\begin{array}{c}\text { Gender } \\
\text { matching }\end{array}$ & $\begin{array}{c}\text { BMI } \\
\text { matching }\end{array}$ & & & \\
\hline $\begin{array}{l}\text { Fayh et al. } \\
\text { (26) }\end{array}$ & $\begin{array}{l}20 \\
(0)\end{array}$ & $\begin{array}{l}10 \\
(0)\end{array}$ & $23.3 \pm 5.5$ & $23.4 \pm 2.6$ & $8.5 \pm 18.8$ & $8.3 \pm 1.3$ & $\begin{array}{l}10.2 \pm 3.4 \\
(F)\end{array}$ & 1 & 1 & 0 & 1 & $\begin{array}{l}\text { MICRO } \\
\text { muscle } \\
\text { (PEAK) }\end{array}$ & $\begin{array}{l}\leftrightarrow \text { Submaximal } \\
\text { aerobic exercise } \\
\text { immediate end } \\
\text { (10\% below } \mathrm{VO}_{2} \\
\text { response at } \\
\text { ventilatory } \\
\text { threshold, } 45 \mathrm{~min} \text { ) } \\
\text { (plethysmography) }\end{array}$ & NA \\
\hline $\begin{array}{l}\text { Franzeck } \\
\text { et al. (27) }\end{array}$ & $\begin{array}{l}8^{*} \\
(12)\end{array}$ & $\begin{array}{l}10^{*} \\
(50)\end{array}$ & $28.5 \pm 5.2$ & $25.1 \pm 1.9$ & $\begin{array}{l}12.0 \pm \\
10.9\end{array}$ & $7.4 \pm 1.3$ & $\begin{array}{l}10.3 \pm 5.2 \\
(\mathrm{NA})\end{array}$ & 0 & 2 & 0 & 0 & $\begin{array}{l}\text { MICRO } \\
\text { cutaneous } \\
\text { (VAR) }\end{array}$ & $\begin{array}{l}\text { FMD (NA) } \\
4 \text { min } \\
\text { (laser Doppler) }\end{array}$ & NA \\
\hline \multirow[t]{2}{*}{ Fujii et al. (28) } & $\begin{array}{l}12 \\
(18)\end{array}$ & $\begin{array}{l}11 \\
(17)\end{array}$ & $25.0 \pm 5.0$ & $24.0 \pm 4.0$ & $12.5 \pm 6.0$ & $7.3 \pm 0.8$ & $\begin{array}{l}\text { NA } \\
\text { (NA) }\end{array}$ & 0 & 1 & 1 & 1 & $\begin{array}{l}\text { MICRO } \\
\text { cutaneous } \\
\text { (NAR) }\end{array}$ & $\begin{array}{l}\leftrightarrow \text { Submaximal } \\
\text { aerobic exercise } \\
\text { immediate end } \\
\left(45 \% \mathrm{VO}_{2 \text { peak }},\right. \\
30 \mathrm{~min})+ \\
\text { Heat } \\
\text { (laser Doppler) }\end{array}$ & $\searrow S N P$ \\
\hline & & & & & & & & & & & & & $\begin{array}{l}\leftrightarrow \text { Submaximal } \\
\text { aerobic exercise } \\
\text { recovery + Heat } \\
\text { (laser Doppler) }\end{array}$ & $\searrow S N P$ \\
\hline $\begin{array}{l}\text { Glowinska- } \\
\text { Olszemska } \\
\text { et al. (29) }\end{array}$ & $\begin{array}{l}52^{\dagger} \\
(54)\end{array}$ & $\begin{array}{l}36^{\dagger} \\
(56)\end{array}$ & $14.5 \pm 2.4$ & $15.1 \pm 2.7$ & $6.0 \pm 3.0$ & $8.7 \pm 1.5$ & $\begin{array}{l}\text { NA } \\
(F)\end{array}$ & 1 & 1 & 2 & 1 & $\begin{array}{l}\text { MACRO } \\
\text { artery } \\
\text { (VAR) }\end{array}$ & $\begin{array}{l}\searrow \text { FMD } \\
4 \text { min on forearm } \\
\text { (ultrasound) }\end{array}$ & NA \\
\hline \multirow[t]{3}{*}{$\begin{array}{l}\text { Gomes et al. } \\
\text { (30) }\end{array}$} & $\begin{array}{l}50^{\dagger} \\
(42)\end{array}$ & $\begin{array}{l}46^{\dagger} \\
(48)\end{array}$ & $32.8 \pm 1.66$ & NA & $15.0 \pm 1.3$ & NA & $\begin{array}{l}\text { NA } \\
\text { (PP) }\end{array}$ & 0 & 2 & 2 & 2 & $\begin{array}{l}\text { MICRO } \\
\text { cutaneous } \\
\text { (PEAK) }\end{array}$ & $\begin{array}{l}\searrow \text { ACh } \\
\text { (laser Doppler) }\end{array}$ & $\searrow S N P$ \\
\hline & & & & & & & & & & & & & $\begin{array}{l}\leftrightarrow \text { PORH (laser } \\
\text { Doppler) }\end{array}$ & NA \\
\hline & & & & & & & & & & & & & $\begin{array}{l}\leftrightarrow \text { Heat (laser } \\
\text { Doppler) }\end{array}$ & NA \\
\hline
\end{tabular}


TABLE 1 | Continued

\begin{tabular}{|c|c|c|c|c|c|}
\hline $\begin{array}{l}\text { Authors and } \\
\text { year of } \\
\text { publication }\end{array}$ & $\begin{array}{c}n \\
\text { (\% women) }\end{array}$ & Age (years) & $\begin{array}{l}\text { Type 1 } \\
\text { diabetes' } \\
\text { duration } \\
\text { (years) }\end{array}$ & $\begin{array}{l}\text { Type } 1 \\
\text { Diabetes } \\
\text { HbA1c } \\
(\%)\end{array}$ & $\begin{array}{l}\text { Glycaemia } \\
\text { before } \\
\text { test } \\
\text { (mmol.L) } \\
\text { (F/PP) }\end{array}$ \\
\hline
\end{tabular}

Quality scores

Vascular

region

Endothelium -

dependent

function (EF)

Endothelium

assessed

independent

(VAR/PEAK) Time and place

unction

of occlusion or
characteristic of

exercise

(technique)

\begin{tabular}{llllllll}
\hline $\begin{array}{l}\text { Type 1 } \\
\text { diabetes }\end{array}$ & HC & $\begin{array}{l}\text { Type 1 } \\
\text { diabetes }\end{array}$ & HC & $\begin{array}{l}\text { Type 1 } \\
\text { diabetes }\end{array}$ & Complications $\begin{array}{c}\text { Age } \\
\text { matching }\end{array}$ & $\begin{array}{c}\text { Gender } \\
\text { matching }\end{array}$ & $\begin{array}{c}\text { BMI } \\
\text { matching }\end{array}$ \\
\hline
\end{tabular}

$\begin{array}{llllllll}\text { Grzelak et al. } & 10 & 21 & 24.3 & 24.2 & \text { NA } & \text { NA } & 6.4 \\ (31)(3 & (0) & (0) & & & & & \text { (NA) }\end{array}$

cohorts)

6.4
(NA)

6.4
(NA)

0

MACR
artery
(2AR)

FMD (NA)

5 min on forearm

(ultrasound)

Intermittent local

exercise

immediate end (30

cycles of exercise:

$\sim 30$ times within

30 s) (NA)

(ultrasound)

\begin{tabular}{|c|c|c|c|c|c|c|c|}
\hline & $\begin{array}{l}10 \\
(0)\end{array}$ & $\begin{array}{l}21 \\
(0)\end{array}$ & 38.6 & 37.7 & NA & NA & $\begin{array}{l}7.2 \\
\text { (NA) }\end{array}$ \\
\hline & $\begin{array}{l}11 \\
(0)\end{array}$ & $\begin{array}{l}29 \\
(0)\end{array}$ & 53.2 & 52.1 & NA & NA & $\begin{array}{l}6.8 \\
\text { (NA) }\end{array}$ \\
\hline $\begin{array}{l}\text { Haak et al. } \\
\text { (32) }\end{array}$ & $\begin{array}{l}9^{\star} \\
(56)\end{array}$ & $\begin{array}{l}9^{*} \\
(45)\end{array}$ & $33.3 \pm 1.0$ & $27.4 \pm 1.1$ & $11.4 \pm 3.0$ & $7.2 \pm 0.2$ & $\begin{array}{l}\text { NA } \\
(\mathrm{F})\end{array}$ \\
\hline $\begin{array}{l}\text { Heier et al. } \\
\text { (33) }\end{array}$ & $\begin{array}{l}46 \\
(48)\end{array}$ & $\begin{array}{l}32 \\
(53)\end{array}$ & $2.0 \pm 0.6$ & $2.2 \pm 0.6$ & 10.0 & $8.7 \pm 1.4$ & $\begin{array}{l}\text { NA } \\
\text { (NA) }\end{array}$ \\
\hline
\end{tabular}

8

NA)

(NA)

MACRO
artery
(VAR)

FMD (NA)

5 min on forearm

(ultrasound)

Intermittent loca

exercise

immediate end (30

cycles of exercise:

$\sim 30$ times within

30 s) (NA)

(ultrasound)

FMD (NA)

5 min on forearm

(ultrasound)

Intermittent local

exercise

immediate end (30

cycles of exercise:

$\sim 30$ times within

30 s) (NA)

(ultrasound)

PORH (NA)

$\begin{array}{ll}\text { cutaneous } & 3 \text { min on arm } \\ \text { (PEAK) } & \text { (capillaroscopy) }\end{array}$

$\mathrm{MICRO} \leftrightarrow \mathrm{PORH}$

cutaneous 5 min on forearm

(VAR) (plethysmography)
NA 
TABLE 1 | Continued

\begin{tabular}{|c|c|c|c|c|c|c|c|c|c|c|c|c|c|c|}
\hline \multirow{2}{*}{$\begin{array}{l}\text { Authors and } \\
\text { year of } \\
\text { publication }\end{array}$} & \multicolumn{2}{|c|}{$\begin{array}{c}n \\
\text { (\% women) }\end{array}$} & \multicolumn{2}{|c|}{ Age (years) } & \multirow{2}{*}{$\begin{array}{l}\text { Type 1 } \\
\text { diabetes' } \\
\text { duration } \\
\text { (years) }\end{array}$} & \multirow{2}{*}{$\begin{array}{l}\text { Type 1 } \\
\text { Diabetes } \\
\text { HbA1c } \\
\text { (\%) }\end{array}$} & \multirow{2}{*}{$\begin{array}{l}\text { Glycaemia } \\
\text { before } \\
\text { test } \\
\text { (mmol.L) } \\
\text { (F/PP) } \\
\\
\begin{array}{l}\text { Type } 1 \\
\text { diabetes }\end{array}\end{array}$} & \multicolumn{4}{|c|}{ Quality scores } & \multirow{2}{*}{$\begin{array}{l}\text { Vascular } \\
\text { region } \\
\text { assessed } \\
\text { (VAR/PEAK) }\end{array}$} & \multirow{2}{*}{$\begin{array}{l}\text { Endothelium- } \\
\text { dependent } \\
\text { function (EF) } \\
\text { Time and place } \\
\text { of occlusion or } \\
\text { characteristic of } \\
\text { exercise } \\
\text { (technique) }\end{array}$} & \multirow{2}{*}{$\begin{array}{l}\text { Endotheliur } \\
\text { independen } \\
\text { function } \\
\text { (VSM) }\end{array}$} \\
\hline & $\begin{array}{l}\text { Type } 1 \\
\text { diabetes }\end{array}$ & $\mathrm{HC}$ & $\begin{array}{l}\text { Type } 1 \\
\text { diabetes }\end{array}$ & $\mathrm{HC}$ & & & & Complications & $\begin{array}{c}\text { Age } \\
\text { matching }\end{array}$ & $\begin{array}{c}\text { Gender } \\
\text { matching }\end{array}$ & $\begin{array}{c}\text { BMI } \\
\text { matching }\end{array}$ & & & \\
\hline $\begin{array}{l}\text { Hoffman et al. } \\
\text { (34) }\end{array}$ & $\begin{array}{l}25 \\
(60)\end{array}$ & $\begin{array}{l}29 \\
(48)\end{array}$ & $15.1 \pm 2.2$ & $14.5 \pm 2.0$ & 5.6 & 7.6 & $\begin{array}{l}\text { NA } \\
\text { (PP) }\end{array}$ & 2 & 2 & 2 & 2 & $\begin{array}{l}\text { MICRO } \\
\text { cutaneous } \\
\text { (PIC) }\end{array}$ & $\begin{array}{l}\leftrightarrow \text { PORH } \\
4 \text { min on arm } \\
\text { (capillaroscopy) }\end{array}$ & NA \\
\hline $\begin{array}{l}\text { Järvisalo et al. } \\
\text { (2) }\end{array}$ & $\begin{array}{l}45^{\dagger} \\
(33)\end{array}$ & $\begin{array}{l}30^{\dagger} \\
(40)\end{array}$ & $11.0 \pm 2.0$ & $11.0 \pm 2.0$ & $4.4 \pm 2.9$ & $8.9 \pm 1.4$ & $\begin{array}{l}12.2 \pm 4.5 \\
(\mathrm{~F})\end{array}$ & 1 & 2 & 2 & 1 & $\begin{array}{l}\text { MACRO } \\
\text { artery } \\
\text { artery } \\
\text { (VAR) }\end{array}$ & $\begin{array}{l}\searrow \text { FMD } \\
4.5 \text { min on forearm } \\
\text { (ultrasound) }\end{array}$ & $\leftrightarrow \mathrm{NMD}$ \\
\hline \multirow[t]{2}{*}{$\begin{array}{l}\text { Johnstone } \\
\text { et al. (35) }\end{array}$} & $\begin{array}{l}15^{*} \\
(73)\end{array}$ & $\begin{array}{l}16^{\star} \\
(75)\end{array}$ & $30.0 \pm 3.9$ & $31.0 \pm 8.0$ & $14.0 \pm 7.7$ & $11.9 \pm 2.3$ & $\begin{array}{l}\text { NA } \\
\text { (PP) }\end{array}$ & 0 & 1 & 1 & 0 & $\begin{array}{l}\text { MICRO } \\
\text { Cutaneous + } \\
\text { muscle } \\
\text { (PEAK) }\end{array}$ & $\begin{array}{l}\searrow \text { MCh } \\
\text { (plethysmography) }\end{array}$ & $\leftrightarrow \mathrm{SNP}$ \\
\hline & & & & & & & & & & & & $\begin{array}{l}\text { MICRO } \\
\text { cutaneous } \\
\text { (PEAK) }\end{array}$ & $\begin{array}{l}\leftrightarrow \text { occlusion- } \\
\text { reperfusion } 5 \text { min } \\
\text { on upper arm } \\
\text { (plethysmography) }\end{array}$ & NA \\
\hline \multirow[t]{2}{*}{$\begin{array}{l}\text { Khan et al. } \\
\text { (36) }\end{array}$} & $\begin{array}{l}55^{*} \\
(59)\end{array}$ & $\begin{array}{l}25 \\
(52)\end{array}$ & $14.8 \pm 3.7$ & $15.4 \pm 4.5$ & $6.6 \pm 4.5$ & $8.7 \pm 1.5$ & $\begin{array}{l}\text { NA } \\
\text { (PP) }\end{array}$ & 0 & 1 & 0 & 1 & $\begin{array}{l}\text { MICRO } \\
\text { cutaneous } \\
\text { (NAR) }\end{array}$ & $\begin{array}{l}\searrow \text { ACh } \\
\text { (laser Doppler) }\end{array}$ & $\searrow S N P$ \\
\hline & & & & & & & & & & & & & $\begin{array}{l}\searrow \text { Heat (laser } \\
\text { Doppler) }\end{array}$ & NA \\
\hline $\begin{array}{l}\text { Koïtka et al. } \\
\text { (37) }\end{array}$ & $\begin{array}{l}12^{\dagger} \\
(50)\end{array}$ & $\begin{array}{l}12^{\dagger} \\
(67)\end{array}$ & $22.0 \pm 3.5$ & $23.0 \pm 3.5$ & $8.9 \pm 6.2$ & $9.2 \pm 2.8$ & $\begin{array}{l}\text { NA } \\
\text { (PP) }\end{array}$ & 0 & 2 & 0 & 1 & $\begin{array}{l}\text { MICRO } \\
\text { cutaneous } \\
\text { (PEAK) }\end{array}$ & $\begin{array}{l}\searrow \text { ACh } \\
\text { (laser Doppler) }\end{array}$ & $\leftrightarrow \mathrm{SNP}$ \\
\hline $\begin{array}{l}\text { Lockhart } \\
\text { et al. (38) }\end{array}$ & $\begin{array}{l}40^{*} \\
(\mathrm{NA})\end{array}$ & $\begin{array}{l}32^{*} \\
(\mathrm{NA})\end{array}$ & $40.0 \pm 12.0$ & $\begin{array}{l}40.4 \pm \\
12.3\end{array}$ & NA & $8.1 \pm 1.2$ & $\begin{array}{l}\text { NA } \\
(F)\end{array}$ & 0 & 2 & 2 & 0 & $\begin{array}{l}\text { MACRO } \\
\text { artery } \\
\text { (VAR) }\end{array}$ & $\begin{array}{l}\searrow \text { FMD } \\
5 \text { min on forearm } \\
\text { (ultrasound) }\end{array}$ & NMD \\
\hline $\begin{array}{l}\text { Lytvyn et al. } \\
\text { (39) }\end{array}$ & $\begin{array}{l}188 \\
\left(51^{\ddagger}\right)\end{array}$ & $\begin{array}{l}65 \\
\left(57^{\ddagger}\right)\end{array}$ & $14.4 \pm 1.7$ & $14.0 \pm 2.0$ & $7.2 \pm 3.2$ & $8.5 \pm 1.3$ & $\begin{array}{l}\text { NA } \\
\text { (NA) }\end{array}$ & 0 & 0 & 0 & 0 & $\begin{array}{l}\text { MACRO } \\
\text { artery } \\
\text { (VAR) }\end{array}$ & $\begin{array}{l}\leftrightarrow \text { FMD } \\
5 \text { min on forearm } \\
\text { (ultrasound) }\end{array}$ & NA \\
\hline $\begin{array}{l}\text { Lytvyn et al. } \\
(40)\end{array}$ & $\begin{array}{l}49 \\
(51)\end{array}$ & $\begin{array}{l}24 \\
(50)\end{array}$ & $26.3 \pm 5.4$ & $25.5 \pm 4.5$ & $14.3 \pm 7.2$ & $7.8 \pm 1.3$ & $\begin{array}{l}\text { NA } \\
\text { (NA) }\end{array}$ & 0 & 0 & 0 & 0 & $\begin{array}{l}\text { MACRO } \\
\text { artery } \\
\text { (VAR) }\end{array}$ & $\begin{array}{l}\text { FMD } \\
\text { (NA) } \\
5 \text { min on forearm } \\
\text { (ultrasound) }\end{array}$ & NMD (NA) \\
\hline
\end{tabular}


TABLE 1 | Continued

\begin{tabular}{|c|c|c|c|c|c|c|c|c|c|c|c|c|c|c|}
\hline \multirow{2}{*}{$\begin{array}{l}\text { Authors and } \\
\text { year of } \\
\text { publication }\end{array}$} & \multicolumn{2}{|c|}{$\begin{array}{c}n \\
\text { (\% women) }\end{array}$} & \multicolumn{2}{|c|}{ Age (years) } & \multirow{2}{*}{$\begin{array}{l}\text { Type 1 } \\
\text { diabetes' } \\
\text { duration } \\
\text { (years) }\end{array}$} & \multirow{2}{*}{$\begin{array}{l}\text { Type } 1 \\
\text { Diabetes } \\
\text { HbA1c } \\
(\%)\end{array}$} & \multirow{2}{*}{$\begin{array}{l}\text { Glycaemia } \\
\text { before } \\
\text { test } \\
\text { (mmol.L) } \\
\text { (F/PP) } \\
\\
\begin{array}{l}\text { Type } 1 \\
\text { diabetes }\end{array}\end{array}$} & \multicolumn{4}{|c|}{ Quality scores } & \multirow{2}{*}{$\begin{array}{l}\text { Vascular } \\
\text { region } \\
\text { assessed } \\
\text { (VAR/PEAK) }\end{array}$} & \multirow{2}{*}{$\begin{array}{l}\text { Endothelium- } \\
\text { dependent } \\
\text { function (EF) } \\
\text { Time and place } \\
\text { of occlusion or } \\
\text { characteristic of } \\
\text { exercise } \\
\text { (technique) }\end{array}$} & \multirow{2}{*}{$\begin{array}{c}\text { Endothelium } \\
\text { independen } \\
\text { function } \\
\text { (VSM) }\end{array}$} \\
\hline & $\begin{array}{l}\text { Type } 1 \\
\text { diabetes }\end{array}$ & $\mathrm{HC}$ & $\begin{array}{l}\text { Type } 1 \\
\text { diabetes }\end{array}$ & $\mathrm{HC}$ & & & & Complications & $\begin{array}{c}\text { Age } \\
\text { matching }\end{array}$ & $\begin{array}{l}\text { Gender } \\
\text { matching }\end{array}$ & $\begin{array}{c}\text { BMI } \\
\text { matching }\end{array}$ & & & \\
\hline $\begin{array}{l}\text { Mackenzie } \\
\text { et al. (41) }\end{array}$ & $\begin{array}{l}122 \\
(43)\end{array}$ & $\begin{array}{l}33 \\
(61)\end{array}$ & $14.1 \pm 2.9$ & $14.2 \pm 3.6$ & $5.3 \pm 3.6$ & $8.7 \pm 1.3$ & $\begin{array}{l}13.4 \pm 4.7 \\
(F)\end{array}$ & 0 & 2 & 2 & 1 & $\begin{array}{l}\text { MACRO } \\
\text { artery } \\
\text { (VAR) }\end{array}$ & $\begin{array}{l}\searrow \text { FMD } \\
4 \text { min on forearm } \\
\text { (ultrasound) }\end{array}$ & $\searrow N M D$ \\
\hline $\begin{array}{l}\text { Maftei et al. } \\
\text { (42) }\end{array}$ & $\begin{array}{l}167 \\
\text { (NA) }\end{array}$ & $\begin{array}{l}57 \\
(\mathrm{NA})\end{array}$ & NA & NA & NA & NA & $\begin{array}{l}\text { NA } \\
\text { (NA) }\end{array}$ & 1 & 2 & 2 & 0 & $\begin{array}{l}\text { MACRO } \\
\text { artery } \\
\text { (VAR) }\end{array}$ & $\begin{array}{l}\searrow F M D \\
5 \text { min on forearm } \\
\text { (ultrasound) }\end{array}$ & $\searrow N M D$ \\
\hline $\begin{array}{l}\text { Mahmud } \\
\text { et al. (3) }\end{array}$ & $\begin{array}{l}20 \\
(40)\end{array}$ & $\begin{array}{l}20 \\
(40)\end{array}$ & $14.2 \pm 1.3$ & $14.1 \pm 1.5$ & NA & $7.5 \pm 1.0$ & $\begin{array}{l}7.4 \pm 3.9 \\
(F)\end{array}$ & 1 & 2 & 2 & 0 & $\begin{array}{l}\text { MICRO } \\
\text { cutaneous } \\
\text { (VAR) }\end{array}$ & $\begin{array}{l}\searrow P O R H \\
5 \text { min on fingers } \\
\text { (tonometry) }\end{array}$ & NA \\
\hline $\begin{array}{l}\text { Mahmud } \\
\text { et al. (43) }\end{array}$ & $\begin{array}{l}23 \\
\left(39^{\ddagger}\right)\end{array}$ & $\begin{array}{l}23 \\
\left(23^{\ddagger}\right)\end{array}$ & $14.6 \pm 1.7$ & $14.7 \pm 1.9$ & $5.8 \pm 3.6$ & $8.3 \pm 1.5$ & $\begin{array}{l}11.1 \pm 5.5 \\
(\mathrm{PP})\end{array}$ & 0 & 2 & 2 & 1 & $\begin{array}{l}\text { MICRO } \\
\text { cutaneous } \\
\text { (VAR) }\end{array}$ & $\begin{array}{l}\searrow P O R H \\
5 \text { min on fingers } \\
\text { (tonometry) }\end{array}$ & NA \\
\hline $\begin{array}{l}\text { Nascimento } \\
\text { et al. (44) }\end{array}$ & $\begin{array}{l}31 \\
\left(39^{\ddagger}\right)\end{array}$ & $\begin{array}{l}58 \\
\left(41^{\ddagger}\right)\end{array}$ & $9.1 \pm 1.8$ & $8.4 \pm 1.8$ & NA & $9.0 \pm 1.6$ & $\begin{array}{l}10.4 \pm 5.5 \\
(\mathrm{PP})\end{array}$ & 1 & 0 & 0 & 0 & $\begin{array}{l}\text { MACRO } \\
\text { artery } \\
\text { (VAR) }\end{array}$ & $\begin{array}{l}\searrow \text { FMD } \\
4 \text { min on forearm } \\
\text { (ultrasound) }\end{array}$ & NA \\
\hline $\begin{array}{l}\text { Palombo } \\
\text { et al. (45) }\end{array}$ & $\begin{array}{l}16 \\
(32)\end{array}$ & $\begin{array}{l}26 \\
(42)\end{array}$ & $18.0 \pm 2.0$ & $19.0 \pm 2.0$ & $11.0 \pm 5.0$ & $7.7 \pm 1.1$ & $\begin{array}{l}9.9 \pm 2.5 \\
(F)\end{array}$ & 1 & 1 & 0 & 1 & $\begin{array}{l}\text { MICRO } \\
\text { cutaneous } \\
\text { (VAR) }\end{array}$ & $\begin{array}{l}\leftrightarrow \mathrm{PORH} \\
5 \text { min on } \\
\text { non-dominant arm } \\
\text { (tonometry) }\end{array}$ & NA \\
\hline $\begin{array}{l}\text { Pareyn et al. } \\
\text { (46) }\end{array}$ & $\begin{array}{l}34 \\
(53)\end{array}$ & $\begin{array}{l}25 \\
(52)\end{array}$ & $15.6 \pm 1.3$ & $15.2 \pm 1.7$ & $6.3 \pm 2.7$ & $8.3 \pm 1.3$ & $\begin{array}{l}10.1 \pm 2.9 \\
(\mathrm{PP})\end{array}$ & 1 & 2 & 2 & 1 & $\begin{array}{l}\text { MICRO } \\
\text { cutaneous } \\
\text { (VAR) }\end{array}$ & $\begin{array}{l}\searrow P O R H \\
5 \text { min on } \\
\text { non-dominant arm } \\
\text { (tonometry) }\end{array}$ & NA \\
\hline $\begin{array}{l}\text { Peltonen } \\
\text { et al. (47) }\end{array}$ & $\begin{array}{l}10 \\
(0)\end{array}$ & $\begin{array}{l}10 \\
(0)\end{array}$ & $33.0 \pm 7.0$ & $32.0 \pm 7.0$ & $11.0 \pm 6.0$ & $7.7 \pm 0.7$ & $\begin{array}{l}9.5 \pm 3.1 \\
(P P)\end{array}$ & 1 & 2 & 0 & 1 & $\begin{array}{l}\text { MICRO } \\
\text { muscle } \\
\text { (NAR) }\end{array}$ & $\begin{array}{l}\searrow \text { During maximal } \\
\text { aerobic exercise } \\
\text { (incremental } 40 \mathrm{~W} \\
3 \mathrm{~min}^{-1} \text { ) (NIRS) }\end{array}$ & NA \\
\hline $\begin{array}{l}\text { Pena et al. } \\
\text { (48) }\end{array}$ & $\begin{array}{l}52 \\
\left(42^{\ddagger}\right)\end{array}$ & $\begin{array}{l}50 \\
\left(50^{\ddagger}\right)\end{array}$ & $14.0 \pm 2.7$ & $14.8 \pm 3.3$ & $5.5 \pm 4.0$ & 8.9 & $\begin{array}{l}11.1 \pm \\
11.1 \pm 4.3 \\
(\mathrm{NA})\end{array}$ & 1 & 2 & 2 & 0 & $\begin{array}{l}\text { MACRO } \\
\text { artery } \\
\text { (VAR) }\end{array}$ & $\begin{array}{l}\searrow F M D \\
4 \text { min on forearm } \\
\text { (ultrasound) }\end{array}$ & $\searrow N M D$ \\
\hline $\begin{array}{l}\text { Pichler et al. } \\
\text { (49) }\end{array}$ & $\begin{array}{l}39 \\
\left(50^{\ddagger}\right)\end{array}$ & $\begin{array}{l}40 \\
\left(40^{\ddagger}\right)\end{array}$ & $12.8 \pm 2.9$ & $12.7 \pm 2.9$ & $4.29 \pm 3.0$ & $9.2 \pm 1.8$ & $\begin{array}{l}12.6 \pm 4.6 \\
(\mathrm{NA})\end{array}$ & 1 & 2 & 2 & 1 & $\begin{array}{l}\text { MICRO } \\
\text { muscle } \\
\text { (PEAK) }\end{array}$ & $\begin{array}{l}\text { \intermittent local } \\
\text { exercise }(60 / \text { min } \\
\text { for } 1 \mathrm{~min}) \text { recovery } \\
\text { (NIRS associated } \\
\text { with venous } \\
\text { occlusion) }\end{array}$ & NA \\
\hline
\end{tabular}


TABLE 1 | Continued

\begin{tabular}{|c|c|c|c|c|c|c|c|c|c|c|c|c|c|c|}
\hline \multirow{2}{*}{$\begin{array}{l}\text { Authors and } \\
\text { year of } \\
\text { publication }\end{array}$} & \multicolumn{2}{|c|}{$\begin{array}{c}n \\
\text { (\% women) }\end{array}$} & \multicolumn{2}{|c|}{ Age (years) } & \multirow{2}{*}{$\begin{array}{l}\text { Type 1 } \\
\text { diabetes' } \\
\text { duration } \\
\text { (years) }\end{array}$} & \multirow{2}{*}{$\begin{array}{l}\text { Type } 1 \\
\text { Diabetes } \\
\text { HbA1c } \\
\text { (\%) }\end{array}$} & \multirow{2}{*}{$\begin{array}{l}\text { Glycaemia } \\
\text { before } \\
\text { test } \\
\text { (mmol.L) } \\
\text { (F/PP) } \\
\\
\begin{array}{l}\text { Type } 1 \\
\text { diabetes }\end{array}\end{array}$} & \multicolumn{4}{|c|}{ Quality scores } & \multirow{2}{*}{$\begin{array}{l}\text { Vascular } \\
\text { region } \\
\text { assessed } \\
\text { (VAR/PEAK) }\end{array}$} & \multirow{2}{*}{$\begin{array}{l}\text { Endothelium- } \\
\text { dependent } \\
\text { function (EF) } \\
\text { Time and place } \\
\text { of occlusion or } \\
\text { characteristic of } \\
\text { exercise } \\
\text { (technique) }\end{array}$} & \multirow{2}{*}{$\begin{array}{l}\text { Endotheliur } \\
\text { independen } \\
\text { function } \\
\text { (VSM) }\end{array}$} \\
\hline & $\begin{array}{l}\text { Type } 1 \\
\text { diabetes }\end{array}$ & $\mathrm{HC}$ & $\begin{array}{l}\text { Type } 1 \\
\text { diabetes }\end{array}$ & $\mathrm{HC}$ & & & & Complications & $\begin{array}{c}\text { Age } \\
\text { matching }\end{array}$ & $\begin{array}{l}\text { Gender } \\
\text { matching }\end{array}$ & $\begin{array}{c}\text { BMI } \\
\text { matching }\end{array}$ & & & \\
\hline $\begin{array}{l}\text { Pillay et al. } \\
\text { (50) }\end{array}$ & $\begin{array}{l}38 \\
\left(58^{\ddagger}\right)\end{array}$ & $\begin{array}{l}28 \\
\left(54^{\ddagger}\right)\end{array}$ & $13.0 \pm 2.9$ & $13.9 \pm 2.7$ & $5.4 \pm 4.6$ & 8.8 & $\begin{array}{l}10.6 \\
10.6 \\
\text { (NA) }\end{array}$ & 0 & 2 & 2 & 0 & $\begin{array}{l}\text { MACRO } \\
\text { artery } \\
\text { (VAR) }\end{array}$ & $\begin{array}{l}\searrow \text { FMD } \\
4 \text { min on forearm } \\
\text { (ultrasound) }\end{array}$ & NA \\
\hline $\begin{array}{l}\text { Rissanen } \\
\text { et al. (51) }\end{array}$ & $\begin{array}{l}7 \\
\text { (0) }\end{array}$ & $\begin{array}{l}10 \\
(0)\end{array}$ & $34.8 \pm 6.0$ & $34.0 \pm 7.0$ & $15.0 \pm 9.0$ & $7.4 \pm 0.9$ & $\begin{array}{l}\text { NA } \\
\text { (PP) }\end{array}$ & 1 & 2 & 1 & 1 & $\begin{array}{l}\text { MICRO } \\
\text { muscle } \\
\text { (VAR) }\end{array}$ & $\begin{array}{l}\searrow \text { maximal aerobic } \\
\text { (incremental } 40 \mathrm{~W} \\
\text { per } 3 \text { min) during } \\
\text { (NIRS) }\end{array}$ & NA \\
\hline \multirow[t]{2}{*}{$\begin{array}{l}\text { Rodriguez- } \\
\text { Manas et al. } \\
\text { (52) (2 } \\
\text { cohorts) }\end{array}$} & $\begin{array}{l}12 \\
\left(25^{\ddagger}\right)\end{array}$ & $\begin{array}{l}14 \\
\left(50^{\ddagger}\right)\end{array}$ & $28.5 \pm 5.9$ & $28.4 \pm 3.4$ & $2.5 \pm 3.8$ & $6.6 \pm 0.8$ & $\begin{array}{l}6.2 \pm 3.5 \\
(\mathrm{NA})\end{array}$ & 1 & 1 & 0 & 1 & $\begin{array}{l}\searrow \text { MICRO } \\
\text { cutaneous + } \\
\text { MICRO } \\
\text { cutaneous + } \\
\text { muscle } \\
\text { (PEAK) }\end{array}$ & $\begin{array}{l}\leftrightarrow \text { MCh } \\
\text { (plethysmography) }\end{array}$ & $\leftrightarrow \mathrm{SNP}$ \\
\hline & $\begin{array}{l}12 \\
(42)\end{array}$ & $\begin{array}{l}14 \\
(50)\end{array}$ & $27.7 \pm 7.6$ & $28.4 \pm 3.4$ & $2.8 \pm 3.46$ & $11.0 \pm 2.3$ & $\begin{array}{l}10.0 \pm 5.2 \\
(N A)\end{array}$ & 1 & 1 & 0 & 1 & $\begin{array}{l}\text { MICRO } \\
\text { cutaneous + } \\
\text { muscle } \\
\text { (PEAK) }\end{array}$ & $\begin{array}{l}\searrow M C h \\
\text { (plethysmography) }\end{array}$ & $\searrow S N P$ \\
\hline $\begin{array}{l}\text { Schlager } \\
\text { et al. (53) }\end{array}$ & $\begin{array}{l}58 \\
(53)\end{array}$ & $\begin{array}{l}58 \\
(41)\end{array}$ & $14.1 \pm 1.7$ & $13.6 \pm 2.0$ & $7.8 \pm 3.3$ & $7.9 \pm 1.0$ & $\begin{array}{l}8.5 \pm 4.7 \\
(\mathrm{NA})\end{array}$ & 1 & 2 & 2 & 0 & $\begin{array}{l}\text { MICRO } \\
\text { cutaneous } \\
\text { (PEAK) }\end{array}$ & $\begin{array}{l}\text { TPORH } \\
3 \text { min (laser } \\
\text { Doppler) }\end{array}$ & NA \\
\hline $\begin{array}{l}\text { Singh et al. } \\
\text { (54) }\end{array}$ & $\begin{array}{l}31 \\
(42)\end{array}$ & $\begin{array}{l}35 \\
(51)\end{array}$ & $15.0 \pm 2.4$ & $15.7 \pm 2.7$ & $6.8 \pm 3.9$ & $8.6 \pm 1.5$ & $\begin{array}{l}8.8 \pm 4.5 \\
(\mathrm{NA})\end{array}$ & 1 & 1 & 1 & 0 & $\begin{array}{l}\text { MACRO } \\
\text { artery } \\
\text { (VAR) }\end{array}$ & $\begin{array}{l}\searrow \text { FMD } \\
5 \text { min on forearm } \\
\text { (ultrasound) }\end{array}$ & $\leftrightarrow \mathrm{NMD}$ \\
\hline $\begin{array}{l}\text { Sochett et al. } \\
\text { (55) }\end{array}$ & $\begin{array}{l}51 \\
\left(51^{\ddagger}\right)\end{array}$ & $\begin{array}{l}59 \\
\left(56^{\ddagger}\right)\end{array}$ & 14.8 & 13.9 & 6.7 & $9.0 \pm 1.0$ & $\begin{array}{l}9.9 \pm 4.5 \\
(\mathrm{NA})\end{array}$ & 1 & 2 & 2 & 0 & $\begin{array}{l}\text { MACRO } \\
\text { artery } \\
\text { (VAR) }\end{array}$ & $\begin{array}{l}\leftrightarrow \text { FMD } \\
5 \text { min on forearm } \\
\text { (ultrasound) }\end{array}$ & NA \\
\hline $\begin{array}{l}\text { Tacito et al. } \\
\text { (56) }\end{array}$ & $\begin{array}{l}32 \\
(63)\end{array}$ & $\begin{array}{l}28 \\
(71)\end{array}$ & $17.3 \pm 4.4$ & $20.1 \pm 5.6$ & $4.1 \pm 2.0$ & $9.95 \pm 3.0$ & $\begin{array}{l}\text { NA } \\
\text { (NA) }\end{array}$ & 0 & 0 & 1 & 1 & $\begin{array}{l}\text { MACRO } \\
\text { artery } \\
\text { (VAR) }\end{array}$ & $\begin{array}{l}\searrow F M D \\
5 \text { min on forearm } \\
\text { (ultrasound) }\end{array}$ & NA \\
\hline $\begin{array}{l}\text { Tagougui } \\
\text { et al. (57) (2 } \\
\text { cohorts) }\end{array}$ & $\begin{array}{l}11^{*} \\
(0)\end{array}$ & $\begin{array}{l}11^{*} \\
(0)\end{array}$ & $27.1 \pm 6.1$ & $25.9 \pm 5.6$ & $4.5 \pm 3.6$ & $6.6 \pm 0.7$ & $\begin{array}{l}\text { NA } \\
\text { (PP) }\end{array}$ & 1 & 2 & 2 & 2 & $\begin{array}{l}\text { MICRO } \\
\text { muscle } \\
\text { (VAR) }\end{array}$ & $\begin{array}{l}\leftrightarrow \text { During maximal } \\
\text { aerobic exercise } \\
\text { (incremental } 20 \mathrm{~W} \\
\text { per } 2 \text { min) } \\
\text { (NIRS) }\end{array}$ & NA \\
\hline
\end{tabular}


TABLE 1 | Continued

$\begin{array}{lcllll}\begin{array}{l}\text { Authors and } \\ \text { year of }\end{array} & n & \text { Age }(\text { years) } & \begin{array}{l}\text { Type 1 } \\ \text { diabetes' }\end{array} & \begin{array}{l}\text { Type 1 } \\ \text { Diabetes }\end{array} & \begin{array}{l}\text { Glycaemia } \\ \text { before }\end{array} \\ \text { publication } & & & \begin{array}{l}\text { duration } \\ \text { (years) }\end{array} & \begin{array}{l}\text { HbA1c } \\ \text { (\%) }\end{array} & \begin{array}{l}\text { test } \\ \text { (mmol.L) }\end{array} \\ & & & & \text { (F/PP) }\end{array}$

Quality scores

Vascular

region

Endothelium

dependent

Endothelium

assessed

function (EF)

independent

(VAR/PEAK) Time and place

of occlusion or

characteristic of

exercise

(technique)

\begin{tabular}{lllllll}
\hline $\begin{array}{l}\text { Type 1 } \\
\text { diabetes }\end{array}$ & HC & $\begin{array}{l}\text { Type 1 } \\
\text { diabetes }\end{array}$ & HC & $\begin{array}{l}\text { Type 1 } \\
\text { diabetes }\end{array}$ & Complications $\begin{array}{c}\text { Age } \\
\text { matching }\end{array}$ & $\begin{array}{c}\text { Gender } \\
\text { matching }\end{array}$ BMI matching \\
\hline
\end{tabular}

$\begin{array}{lllllll}12^{*} & 12^{\star} & 25.5 \pm 7.3 & 26.2 \pm 5.0 & 10.9 \pm 3.4 & 9.1 \pm 0.7 & \text { NA }\end{array}$

MICRO

$\searrow$ During maximal

muscle aerobic exercise

(incremental 20 W

per $2 \mathrm{~min}$ )

(NIRS)

$\begin{array}{llllllll}\text { Tibiriça et al. } & 48^{*} & 34^{*} & \text { NA } & \text { NA } & \text { NA } & 9.7 \pm 2.5 & 10.5 \pm 5.7 \\ (58) & (58) & (53) & & & & & \text { (PP) }\end{array}$

2

Oral contraceptive

5

$7.7 \pm 0.75 \quad 9.3 \pm 4.8$
Waclawovsky 14
$30.3 \pm 6.0$
$26.8 \pm 5.1 \quad N A$
(PP)
$\begin{array}{ll}\text { Waclawovsky } & \text { (0) } \\ \text { et al. (60) } & \end{array}$ 
TABLE 1 | Continued

\begin{tabular}{|c|c|c|c|c|c|c|c|c|c|c|c|c|c|c|}
\hline \multirow{2}{*}{$\begin{array}{l}\text { Authors and } \\
\text { year of } \\
\text { publication }\end{array}$} & \multicolumn{2}{|c|}{$\begin{array}{c}n \\
\text { (\% women) }\end{array}$} & \multicolumn{2}{|c|}{ Age (years) } & \multirow{2}{*}{$\begin{array}{l}\text { Type } 1 \\
\text { diabetes' } \\
\text { duration } \\
\text { (years) }\end{array}$} & \multirow{2}{*}{$\begin{array}{l}\text { Type 1 } \\
\text { Diabetes } \\
\text { HbA1c } \\
\text { (\%) }\end{array}$} & \multirow{2}{*}{$\begin{array}{l}\text { Glycaemia } \\
\text { before } \\
\text { test } \\
\text { (mmol.L) } \\
\text { (F/PP) } \\
\\
\begin{array}{l}\text { Type } 1 \\
\text { diabetes }\end{array}\end{array}$} & \multicolumn{4}{|c|}{ Quality scores } & \multirow{2}{*}{$\begin{array}{l}\text { Vascular } \\
\text { region } \\
\text { assessed } \\
\text { (VAR/PEAK) }\end{array}$} & \multirow{2}{*}{$\begin{array}{l}\text { Endothelium- } \\
\text { dependent } \\
\text { function (EF) } \\
\text { Time and place } \\
\text { of occlusion or } \\
\text { characteristic of } \\
\text { exercise } \\
\text { (technique) }\end{array}$} & \multirow{2}{*}{$\begin{array}{l}\text { Endothelium } \\
\text { independent } \\
\text { function } \\
\text { (VSM) }\end{array}$} \\
\hline & $\begin{array}{l}\text { Type } 1 \\
\text { diabetes }\end{array}$ & $\mathrm{HC}$ & $\begin{array}{l}\text { Type } 1 \\
\text { diabetes }\end{array}$ & $\mathrm{HC}$ & & & & Complications & $\begin{array}{c}\text { Age } \\
\text { matching }\end{array}$ & $\begin{array}{l}\text { Gender } \\
\text { matching }\end{array}$ & $\begin{array}{c}\text { BMI } \\
\text { matching }\end{array}$ & & & \\
\hline $\begin{array}{l}\text { Waring et al. } \\
\text { (61) }\end{array}$ & $\begin{array}{l}8 \\
(0)\end{array}$ & $\begin{array}{l}8 \\
\text { (0) }\end{array}$ & $30.0 \pm 5.7$ & $30.0 \pm 5.7$ & NA & NA & $\begin{array}{l}8.5 \pm 3.1 \\
(\mathrm{NA})\end{array}$ & 0 & 2 & 1 & 1 & $\begin{array}{l}\text { MICRO } \\
\text { cutaneous + } \\
\text { muscle } \\
\text { (PEAK) }\end{array}$ & $\begin{array}{l}\searrow \text { Ach } \\
\text { (plethysmography) }\end{array}$ & $\leftrightarrow \mathrm{SNP}$ \\
\hline $\begin{array}{l}\text { Wiltshire et al. } \\
\text { (62) }\end{array}$ & $\begin{array}{l}35 \\
(49)\end{array}$ & $\begin{array}{l}20 \\
(50)\end{array}$ & $13.7 \pm 2.2$ & $13.8 \pm 2.5$ & $5.7 \pm 3.3$ & $9.1 \pm 0.9$ & $\begin{array}{l}\text { NA } \\
\text { (NA) }\end{array}$ & 1 & 2 & 2 & 0 & $\begin{array}{l}\text { MACRO } \\
\text { artery } \\
\text { (VAR) }\end{array}$ & $\begin{array}{l}\searrow \text { FMD } \\
4.5 \text { min on forearm } \\
\text { (ultrasound) }\end{array}$ & $\leftrightarrow \mathrm{NMD}$ \\
\hline $\begin{array}{l}\text { Wotherspoon } \\
\text { et al. (63) }\end{array}$ & $\begin{array}{l}15^{*} \\
(27)\end{array}$ & $\begin{array}{l}15^{\star} \\
(40)\end{array}$ & $39.7 \pm 10.1$ & $35.8 \pm 9.7$ & $\begin{array}{l}20.6 \pm \\
11.8\end{array}$ & $7.9 \pm 0.8$ & $\begin{array}{l}12.1 \pm 6.4 \\
(\mathrm{NA})\end{array}$ & 1 & 1 & 0 & 1 & $\begin{array}{l}\text { MICRO } \\
\text { cutaneous + } \\
\text { muscle } \\
\text { (PEAK) }\end{array}$ & $\begin{array}{l}\leftrightarrow \text { ACh } \\
\text { (plethysmography) }\end{array}$ & $\leftrightarrow \mathrm{SNP}$ \\
\hline $\begin{array}{l}\text { Yazici et al. } \\
\text { (64) }\end{array}$ & $\begin{array}{l}30 \\
(60)\end{array}$ & $\begin{array}{l}29 \\
(55)\end{array}$ & $29.0 \pm 6.0$ & $30.0 \pm 6.0$ & $\begin{array}{l}7.79 \pm \\
5.79\end{array}$ & $7.7 \pm 1.31$ & $\begin{array}{l}8.0 \pm 3.1 \\
(F)\end{array}$ & 1 & 2 & 2 & 0 & $\begin{array}{l}\text { MACRO } \\
\text { artery } \\
\text { (VAR) }\end{array}$ & $\begin{array}{l}\searrow \text { FMD } \\
5 \text { min on forearm } \\
\text { (ultrasound) }\end{array}$ & $\leftrightarrow \mathrm{NMD}$ \\
\hline
\end{tabular}

Mean \pm SD; HC, healthy controls; \the outcome was significantly lower in the group with type 1 diabetes vs. HC group; $\leftrightarrow$ the outcome was not significantly $(P>0.05)$ different between the two groups; $\uparrow$ the outcome was significantly higher in the group with type 1 diabetes vs. HC group.

In italics: the measurement' technique.

Quality scores: complications 1: the absence of complication was validated (no retinopathy, no nephropathy, no neuropathy), 0: one of the three complications was not noted in the article and the authors did not respond to further inquiry. Matching 2: noticed in article, 1: visibly correct, 0: not noted in the article.

MICRO, means the study focused on the microcirculation; MACRO, means the study focused on macrocirculation.

PEAK, Data displaved as peak values in the original paper; VAR, Data displayed as variations from baseline in the original paper.

Occlusion-reperfusion stimuli FMD, Flow-Mediated Dilation; PORH, Post-Occlusive Reactive Hyperaemia; Pharmacological stimuli NMD, Nitroglycerin-Mediated Dilation; ACh, Acetylcholine; MCh, Methacholine.

${ }^{*}$ means that smokers were included; ${ }^{\dagger}$ means that no information on smoking was provided in the article.

Fmeans that none of the included women was taking oral contraceptives.

F, vascular measurements were performed during fasting; PP, vascular measurements were conducted at post-prandial state.

Overall, the 58 studies on endothelial function involved 15-377 participants, with a mean age ranging from 8.0 to 52.7 years, mean BMI from 18.4 to $26.6 \mathrm{~kg} . \mathrm{m}^{-2}$. mean SBP from 93.9 to $126.5 \mathrm{mmHg}$. mean DBP from 57.4 to 82.5 $\mathrm{mmHg}$, and mean cholesterol, $\mathrm{HDL}$-cholesterol and triglycerides from 3.7 to $5.3 \mathrm{mmol}_{\mathrm{L}} \mathrm{L}^{-1}$, from 1.1 to $1.8 \mathrm{mmol} . \mathrm{L}^{-1}$ and from 0.7 to $3.3 \mathrm{mmol} . \mathrm{L}^{-1}$, respectively. Among participants with type 1 diabetes, mean $\mathrm{Hb} \mathrm{A}_{1 \mathrm{c}}$ and mean diabetes duration ranged from 6.6 to $11.9 \%$ (from 7.0 to $14.9 \mathrm{mmol} . \mathrm{L}^{-1}$ ) and from 2.5 to 21.1 years, respectively. 
a selection was performed thanks to the eligibility criteria, as described below. In the case of unclear or missing information, the authors were contacted for clarification.

\section{Study Selection}

The main inclusion criteria were having assessed the peripheral vasodilatory capacity (dependent or not on endothelial function, i.e., corresponding to either endothelium or VSM function) in humans (men and/or women, no age limit) with type 1 diabetes free from micro- and/or macro-vascular complications ("P" from the PICOS) compared with healthy controls ("C" from the PICOS; i.e., case control studies, "S" from the PICOS). The absence of micro (retinopathy, nephropathy-i.e., albuminuria $>40 \mathrm{mg} \cdot \mathrm{dL}^{-1}$, neuropathy) and macro- (cardiac, peripheral, and cerebral) vessels complications in groups of patients with type 1 diabetes was checked based on the B category of the DCCT (exclusion of the $\mathrm{C}$ category).

Studies dealing with vasoreactivity in brain, heart, or retina were excluded because cerebral and cardiac vessels constitute a specific system and, contrary to peripheral vessels, the retinal vasculature is highly differentiated. Articles using needle injury methods were excluded because of additional contributions of the nervous system $(21,40)$. Only the studies using the following stimuli for vascular function assessment were selected: postocclusion reactive hyperemia, local heat, physical exercise, and pharmacological substances ("I" from the PICOS). Sometimes, two stimuli were applied at the same time $(12,28,59)$. In cases of interventional studies testing supplementations (32, 40, 61), drugs (61), or in a cohort study (19) only baseline values, i.e., before intervention, were analyzed in the meta-analyses.

Where the same data appeared in several publications by the same (or part of the same) group of subjects $(58,59,63,70-72)$, we opted to include the primary or the most exhaustive article (Table 1). Study selections were undertaken independently by two investigators (EL and $\mathrm{EH}$ ). In the case of disagreements on eligibility criteria, the discrepancies were resolved by consensus with a third reviewer $(\mathrm{PF})$.

\section{Data Extraction}

The absence of vascular complications in clinical stage was carefully checked. We assessed the quality of populations matching by age, gender, and BMI (Table 1). When the authors provided only the standard error (SE) and did not respond to our request to provide standard deviations (SD), we calculated the corresponding SD, assuming the data was normally distributed.

Vascular outcomes reported corresponded either to the peak response or to a variation from baseline to peak ("O" from the PICOS). Where only peak values were provided ( $c f$. in 18 studies, Table 1), we contacted the authors to calculate the corresponding variation. These data obtained from eight studies $(26,28,46,47$, $49,51,59,60)$ were then used in an additional analysis where only variations (either initially found in the papers or a posteriori calculated) were tested.

\section{Data Analysis}

For statistical analyses we used the standardized mean difference (SMD) (Cohen's d) of endothelial or non-endothelial-dependent vascular function between the type 1 diabetes and the healthy control group. SMD allowed to standardize micro- and macrocirculation assessments as well as the large panel of measurement techniques used. Negative SMD corresponded to impaired vascular function.

We calculated weighted pooled summary estimates of SMD. For each meta-analysis, we used the DerSimonian and Laird method. Accordingly, studies were considered to be a random sample from a population of studies. Heterogeneity was assessed using $I^{2}$ and chi-square heterogeneity statistics. A randomeffects model was used to combine data. The overall effect was estimated using a weighted average of individual effects, with weights inversely proportional to variance in observed effects. Heterogeneity between studies was quantified using $I^{2}$ statistics, with upper limits of 25,50 , and $75 \%$ as low, moderate, and high, respectively (73). Publication bias was evaluated with funnel plot and Egger's test. The pooled SMD were estimated with $95 \%$ confidence interval (CI). All analyses were performed using $\mathrm{R}$ software with the survival and metafor packages.

\section{Main Meta-Analyses (Primary Objective)}

First, two meta-analyses, for endothelial function and vascular smooth muscle function, were conducted including all studies, regardless of the stimulus used or the vascular bed explored. As some studies explored the effects of more than one stimulus successively, or used more than one pharmacological substance, and some studies assessed several periods surrounding exercise (Table 1), a priority order was established (Supplementary Table S1). Overall, the stimuli inducing vasodilatation were ordered from the most physiological to the least physiological (exercise, occlusion-reperfusion, heat, and then pharmacological substances).

Exercise stimuli were either aerobic (varying from 20 to $40 \mathrm{~min}$ and from $45 \% \mathrm{VO}_{2 \text { peak }}$ to $100 \% \mathrm{VO}_{2 \max }$ ) or local intermittent concentric/eccentric handgrip (duration from $30 \mathrm{~s}$ to $3 \mathrm{~min}$; Table 1). Only one paper used two types of exercise, i.e., a session of resistance exercise $(40 \mathrm{~min})$ and aerobic exercise (40 $\min )(60)$.

\section{Metaregression Analyses (Sensitivity Analyses)}

In order to disentangle, which of the many factors contributes most to the development and/or manifestation of the vascular dysfunction possibly observed in main meta-analyses, we performed metaregression analyses.

The influence of qualitative outcomes [i.e., study design (vessels size, vascular region, stimuli, technique, and types of exercise) and some subjects' characteristics (gender, generation), see Supplementary Table S2 for SMD of endothelial function and Supplementary Table S4 for SMD of VSM function] on vascular functions SMD were analyzed by comparing subgroups of studies. When a study could be included in two subgroups of the metaregression (e.g., a study analyzing separately both exercise and occlusion reperfusion stimuli in the same population), it was inserted only into the subgroup with the higher order of priority. 
The influence of quantitative outcomes [i.e., the other studies' characteristics (sample size) and participant characteristics (mean age, BMI among subjects' with type 1 diabetes), and presence of concomitant risk factors (mean $\mathrm{HbA}_{1 \mathrm{c}}$ and duration of disease in patients; difference between patients and healthy controls in mean BMI, SBP, DBP, total cholesterol, HDLcholesterol, and triglycerides; SMD in patients vs. controls of blood flow and diameter baseline values)] were tested using univariate metaregression analyses.

\section{RESULTS}

\section{Study Selection and Characteristics}

The flow diagram (Figure 1) describes the criteria followed in order to select articles for inclusion in this meta-analysis, and 9,232 studies were identified in the first stage. After removing duplicates and out-of-scope studies, 1,826 studies were screened of which 92 articles met the inclusion criteria. Thirty-four studies were excluded after screening the abstract or reading the entire methods section. Ultimately, 58 studies among which 3 involved two $(52,57)$ or three $(31)$ cohorts of patients vs. controls (in 2,322 subjects with type 1 diabetes and 1,777 healthy controls) assessing the endothelial function and 21 studies among which 1 involved 2 cohorts (52) (in 916 subjects with type 1 diabetes and 553 healthy controls) focusing on the VSM were included in the meta-analysis. The studies which measured VSM also assessed endothelial function. The studies main characteristics are reported in Table $\mathbf{1}$.

\section{Primary Outcomes (Main Meta-Analyses)}

The endothelial function meta-analysis (micro- and macrocirculation pooled) revealed a significant impairment in patients with type 1 diabetes compared to healthy controls (SMD = $-0.61 ; 95 \% \mathrm{CI}=-0.79,-0.44,4,099$ subjects, $P<0.001)$, albeit with a great heterogeneity $\left(I^{2}=85.7 \%, P<0.001\right.$; forest plot in Figure 2 and funnel plot in Supplementary Figure S1). Comparable results was obtained when only endothelial function calculated as a variation from baseline were included ( $c f$ data displayed in the original papers for 43 studies and a posteriori calculated variations for 3 additional studies) ( $\mathrm{SMD}=-0.69$; 95\% CI $=-0.88,-0.50 ; P<0.001)$.

The non-endothelial (i.e., VSM) function meta-analysis demonstrated a significant impairment in type 1 diabetes compared to the control group $(1,469$ subjects, $P<0.05$; forest plot in Figure 6 and funnel plot in Supplementary Figure S2), with significant heterogeneity $\left(I^{2}=78.6 \%, P<0.001\right)$.

Since we found some evidence of high heterogeneity, metaregressions as well as subgroup analyses were performed to determine the sources of heterogeneity. The metaregressions were also intended to explore potential moderating factors of endothelial or VSM dysfunction.

\section{Metaregressions (Sensitivity Analyses) for Endothelial Function}

The endothelial function impairment in type 1 diabetes affected both the macrocirculation and the microcirculation (28 and 30 studies with 2,929 and 1,343 subjects, respectively; Figure 2).
Macrovessels appeared to be the most affected when considering the entire population (moderator $P=0.001$, Figures $2 \mathbf{A}, \mathbf{B}$ ) or adults separately (nine studies, SMD $=-1.06$; $95 \% \mathrm{CI}=-1.51$, -0.60 for macrocirculation vs. 22 studies, $\mathrm{SMD}=-0.24 ; 95 \% \mathrm{CI}$ $=-0.51,0.01$ for microcirculation, $P<0.01$ ).

In line with the more marked alteration in macrovascular vs. microvascular beds, endothelial dysfunction associated with diabetes was greater in artery compared to capillaries (Figure 3). We performed additional analyses on factors which may have contributed to this pronounced difference between macro- and micro-circulation. Noteworthy, there was an overall significant moderating effect of techniques used, translating in posthoc analyses into a greater impairment of type 1 diabetes endothelial function when measured by the gold standard macrovascular technique "ultrasound" (28 studies on artery) compared to plethysmography (11 studies on cutaneous vessels; Figure 4).

The stimulus used did not seem to have a significant impact on the findings. Lower basal artery diameter in type 1 diabetes compared to controls aggravated endothelial (FMD) dysfunction $(\beta=1.70 ; P<0.001)$.

Focusing on exercise and its characteristics, impairment of vasodilatory capacity was more noticeable when regional isometric exercise (intermittent handgrip, 2 studies whose 1 including 3 cohorts) was used, compared to general aerobic exercise (constant-load for 1 study and incremental for three studies; Figure 5).

Metaregressions based on other qualitative outcomes did not show any significant results (Supplementary Tables S2, S3).

When taking into account the demographic or disease characteristics of the patients included, higher $\mathrm{HbA}_{1 c}$ levels in patients with type 1 diabetes were associated with more pronounced endothelial dysfunction in the whole population $(\beta$ $=-0.20 ; P<0.05)$ or when focusing only on children and adolescents $(\beta=-0.43 ; P<0.01)$.

We further examined the potential impact of concomitant risk factors differences between patients and healthy controls. Surprisingly, the difference in mean BMI between children/adolescents with type 1 diabetes and healthy controls was positively associated with endothelial function (i.e., the less BMI those in type 1 diabetes were higher, the better was the endothelial function; $\beta=0.39, P<0.001$ ), but with median values of mean BMI still corresponding to normal weight in these young subjects with type 1 diabetes (i.e., $20.9 \mathrm{~kg} . \mathrm{m}^{-2}$ ). This moderating impact of BMI on endothelial dysfunction was also found in children and adolescents and in adults with type 1 diabetes when analyzing only the macrocirculation (data not shown). No other metaregression with studies' characteristics (including sample size), participant characteristics, or presence of concomitant risk factors, was significant.

\section{Subgroup Analyses for Endothelial Function}

Considering the high heterogeneity, we have taken the care to analyze also the effect of each intervention (i.e., exercise and FMD in large vessels; exercise, heat, pharmacological substances 

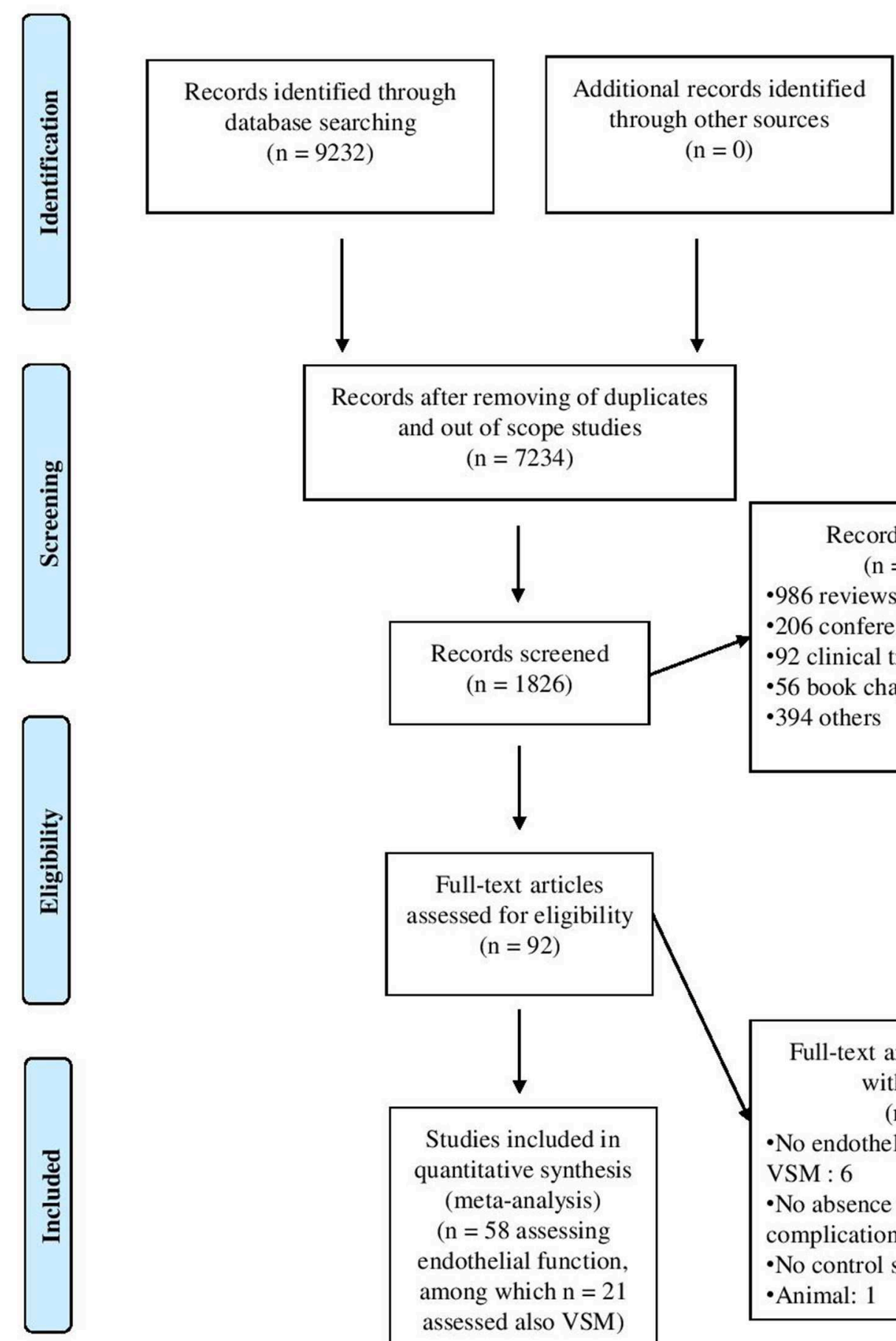

Records excluded

$(n=1734)$

.986 reviews

-206 conferences

.92 clinical trials abstracts

- 56 book chapters

-394 others

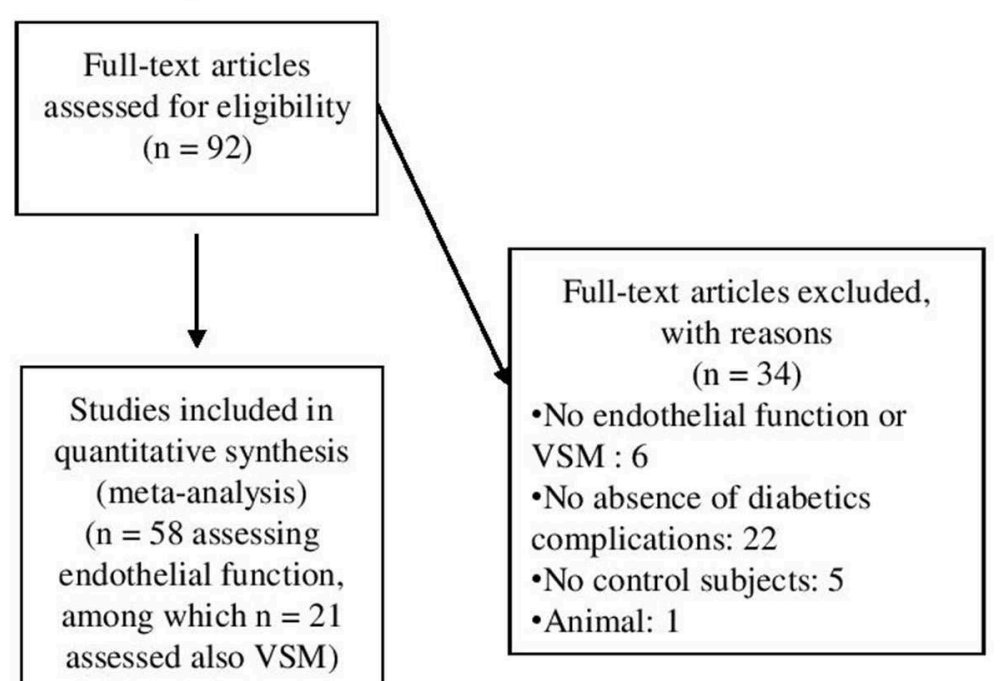

FIGURE 1 | Flow-chart outlining the process of study selection.

and PORH in small vessels) separately in Figures 2A,B. For large vessels, the endothelial function, as assessed in response to exercise and FMD separately, was significantly impaired in patients with type 1 diabetes for both stimuli $(P<0.01$ and $P<0.001$, respectively; Figure 2A). For small vessels however, the effects of exercise and pharmacological substances separately did not reach statistical significance $(P=0.32$, and $P=$ 0.52 , respectively), while endothelial dysfunction was visible in response to heat and PORH $(P<0.01$ and $P<0.05$, respectively).

For each stimulus in microvessels, we also analyzed separately each technique of measure (Supplementary Figure S3). For all stimuli in macrovessels (i.e., exercise and FMD) only the 
technique of ultrasounds was used. Overall, the heterogeneity remained high despite these subdivisions. However, focusing specifically on only one type of exercise, i.e., local intermittent exercise, allowed to reduce heterogeneity to a level close to moderate.

\section{Metaregressions (Sensitivity Analyses) for VSM Function}

Macrocirculation (i.e., brachial artery in all studies; 1,151 subjects) and microcirculation (i.e., subcutaneous capillaries in all studies; 348 subjects) VSM function was altered to the same extent in the whole population (Figure 6) as well as when focusing separately on adults or children/adolescents (data not shown). Other metaregressions based on qualitative outcomes were not significant (Supplementary Table S4).

Higher triglycerides in patients with type 1 diabetes vs. controls were determinants of a more altered VSM (metaregression between difference in mean triglycerides and VSM SMD, $\beta=-0.80 ; P<0.05)$. When focusing only on the macrovascular bed, difference in mean SBP and DBP were inversely associated with VSM SMD $(\beta=-0.13, P<0.001$ and $\beta=-0.20, P<0.01$, respectively). In the microvasculature, the numbers of studies focusing only on children and adolescents $(n=1)$, or taking into account HDL-C $(n=2)$ or triglycerides $(n=2)$ were too small for performing subgroup analyses using these outcomes. No other subgroup or metaregressions analyses for VSM SMD were significant.

\section{Comparison Between Endothelial and VSM Dysfunction}

In 12 of the 21 studies where both endothelial function and VSM were measured, authors found a significant impairment only in endothelial function (Table 1). This result is in accordance with sub-analyses revealing that endothelial function (58 studies) tended to be more affected compared to VSM (21 studies; $P=0.08$ ).

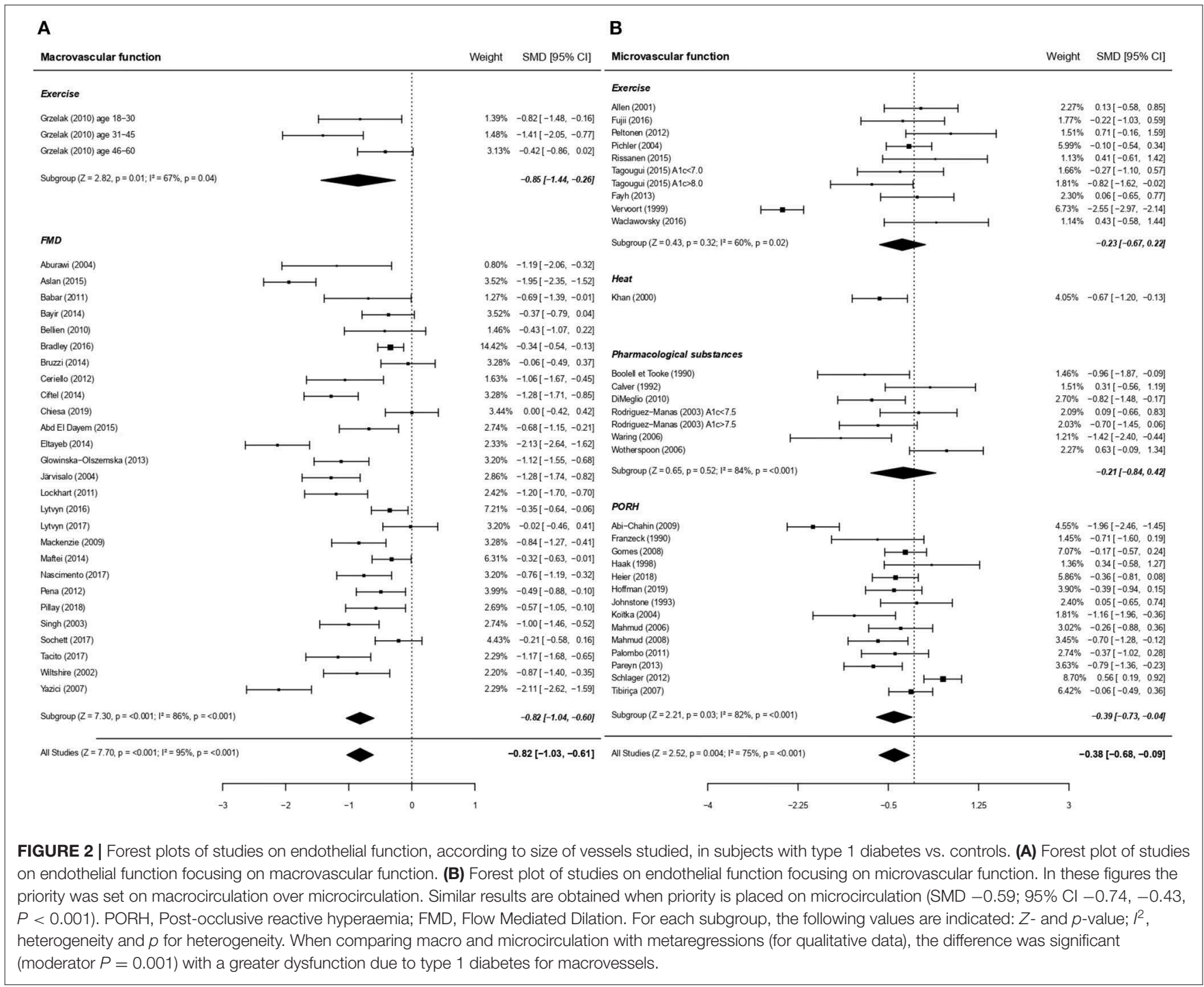




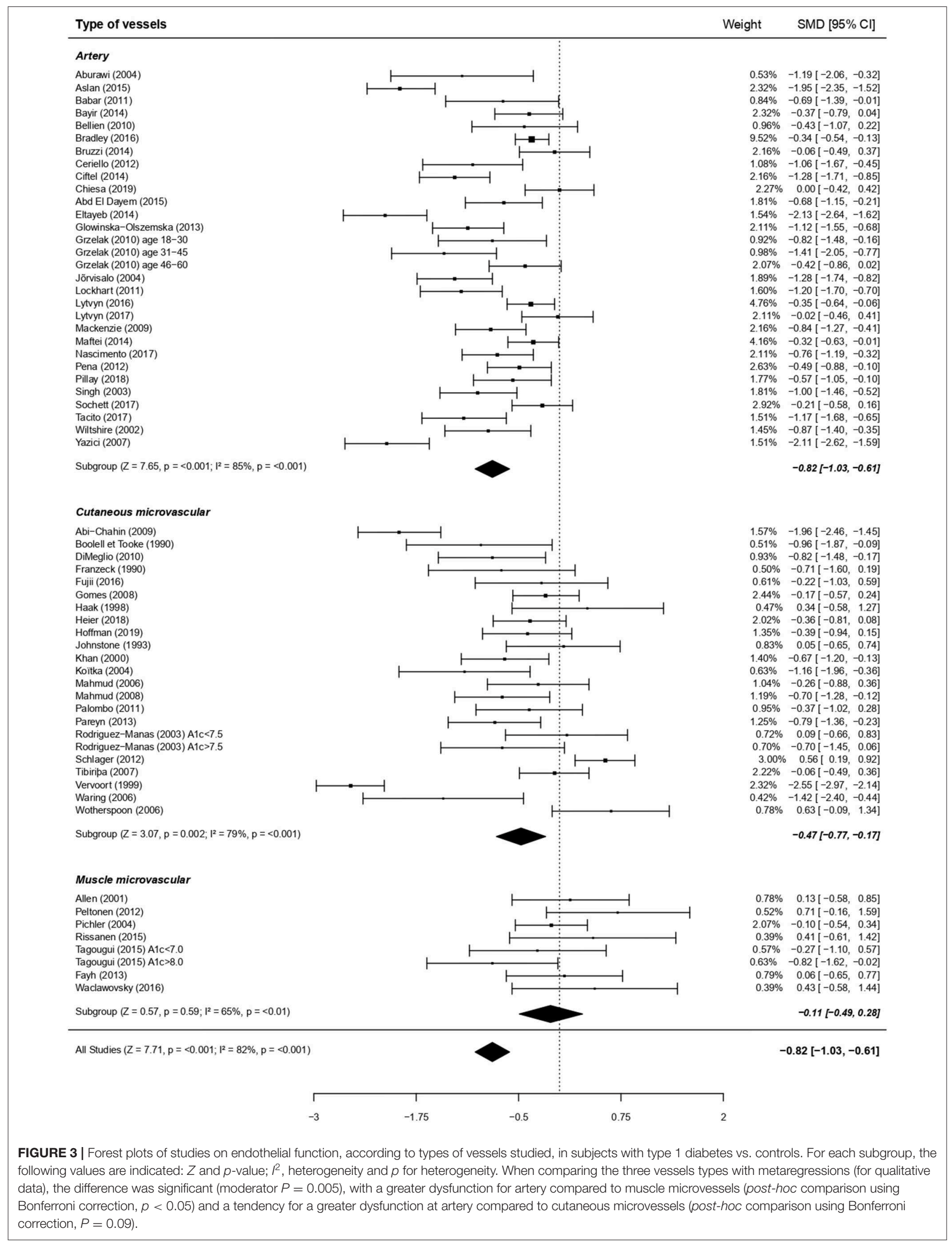


Measurement techniques

Weight SMD $[95 \% \mathrm{Cl}]$

\section{Capillaroscopy}

Abi-Chahin (2009)

Haak (1998)

Tibiriça (2007)

Subgroup $\left(Z=7.65, p=0.28 ;\left.\right|^{2}=85 \%, p=<0.001\right)$

\section{Laser Doppler}

Boolell et Tooke (1990)

DiMeglio (2010)

Franzeck (1990)

Fujii (2016)

Khan (2000)

Khan $(2000)$
Koïtka (2004)

Schlager (2012)

Subgroup $\left(Z=1.88, p=0.05 ;\left.\right|^{2}=79 \%, p=<0.001\right)$

\section{NIRS}

Peltonen (2012)

Pichler (2004)

Tagougui (2015) A1c $<7.0$

Tagougui (2015) A1c $>8.0$

Subgroup $\left(Z=0.68, p=0.49 ; l^{2}=65 \%, p=0.02\right)$

\section{Plethysmography}

Allen (2001)

Calver (1992)

Johnstone (1993)

Rodriguez-Manas (2003) A1c $<7.5$

Rodriguez-Manas (2003) A1c $>7.5$

Fayh (2013)

Vervoort (1999)

Waclawovsky (2016)

Waring (2006)

Subgroup $\left(Z=0.61, p=0.54 ;\left.\right|^{2}=60 \%, p=0.01\right)$

Tonometry

Mahmud (2006)
Mahmud (2008)

Palombo (2011)

Palombo (2011)
Pareyn (2013)

Subgroup $\left(Z=3.60, p=<0.001 ; 1^{2}=0 \%, p=0.55\right.$ )

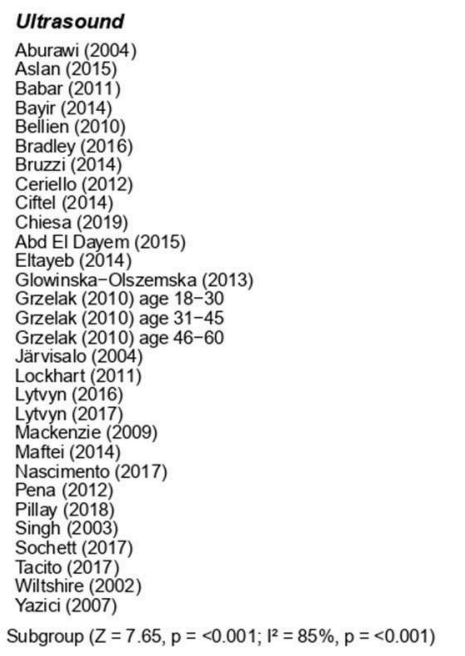

Ultrasound

Aslan (2015)

Babar (2011)

Bayir (2014)

Bradley (2016)

Ciftel (2014)

Abd El Dayem (2015)

Lytryn (2016)

Mackenzie (2009)

ascimento (2017)

ena (2012)

ingh (2003)

Tachett (2017)

Yazici (2007)

Subgroup $\left(Z=7.65, p=<0.001 ;\left.\right|^{2}=85 \%, p=<0.001\right)$
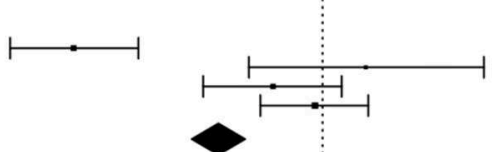

$1.56 \%-1.96[-2.46,-1.45]$

$0.47 \% \quad 0.34[-0.58, \quad 1.27$

$2.20 \%-0.06[-0.49,0.36]$

$-0.82[-1.03,-0.61]$

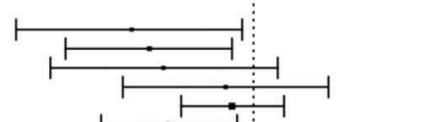

$0.50 \%-0.96[-1.87,-0.09$

$0.93 \%-0.82[-1.48,-0.17$

$0.61 \%-0.22[-1.03,0.59$

$2.61 \% \%-0.17[-0.57,0.54$

$139 \%-0.67[-120,-0.13$

$0.62 \%-1.16[-1.96-0.36$

$2.99 \% \quad 0.56[0.19,0.92$

$-0.47[-0.96,-0.10]$

$0.52 \% \quad 0.71[-0.16, \quad 1.59]$

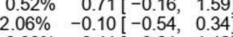

$0.39 \% \quad 0.41[-0.61,1.42]$

$0.57 \%-0.27[-1.10, \quad 0.57]$

$-0.20[-0.78,0.37]$

$2.01 \%-0.36[-0.81,0.08$

$0.82 \% \quad 0.05[-0.65,0.74$

$0.72 \% \quad 0.09[-0.66,0.83]$

$0.70 \%-0.70[-1.45, \quad 0.06$

$2.79 \% \quad 0.06[-0.65,0.77$

$2.31 \%-2.55[-2.97,-2.14$

$0.41 \%-1.42[-2.50,-0.44]$

$0.78 \% \quad 0.63[-0.09,1.34]$

$-0.10[-0.42,0.22]$

$\begin{array}{lll}1.04 \% & -0.26[-0.88, & 0.36 \\ 1.18 \% & -0.70[-1.28, & -0.12]\end{array}$

$.18 \%-0.70[-1.28,-0.12$

$1.25 \%-0.79[-1.36,-0.23]$

$-0.55[-0.85,-0.25]$

$0.53 \%-1.19[-2.06,-0.32]$

$2.31 \%-1.95[-2.35,-1.52]$

$2.31 \%-0.37[-0.79,0.04]$

$2.15 \%-0.06[-0.49,0.37$

$1.07 \%-1.06[-1.67,-0.45$

$2.15 \%-1.28[-1.71,-0.85$

$2.26 \% 0.00[-0.42,0.42$

$1.80 \%-0.68[-1.15,-0.21$

$1.53 \%-2.13[-2.64,-1.62]$

$2.10 \%-1.12[-1.55,-0.68]$

$0.9 \%-1.41[-2.05,-0.77$

$206 \%-0.42[-0.86,0.02$

$1.88 \%-1.28[-1.74,-0.82$

$1.59 \%-1.20[-1.70-0.70$

$4.73 \%-0.35[-0.64,-0.06$

$2.10 \%-0.02[-0.46,0.41$

$2.15 \%-0.84[-1.27,-0.41$

$4.14 \%-0.32[-0.63,-0.01]$

$2.10 \%-0.76[-1.19,-0.32$

$2.62 \%-0.49(-0.88,-0.10)$

$1.76 \%-0.57[-1.05,-0.10$

$2.91 \%-0.21[-0.58,0.16$

$150 \%-1.17[-1.68,-0.65$

$1.44 \%-0.87[-1.40,-0.35$

$1.50 \%-2.11[-2.62,-1.59]$

$-0.82[-1.03,-0.61]$

$-0.59[-0.74,-0.43]$

All Studies $\left(Z=7.71, p=<0.001 ; I^{2}=82 \%, p=<0.001\right)$
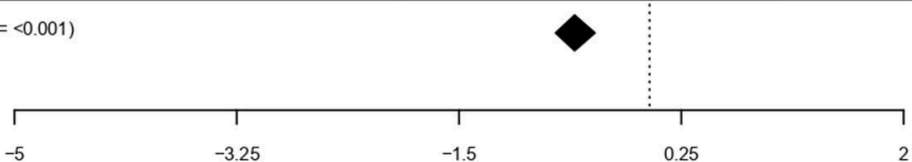

FIGURE 4 | Forest plots of studies on endothelial function, according to measurement techniques, in subjects with type 1 diabetes vs. controls. For each subgroup, the following values are indicated: $Z$ and $p$-value; $l^{2}$, heterogeneity and $p$ for heterogeneity. When comparing the six types of techniques with metaregressions (for qualitative data), the difference was significant (moderator $P=0.02$ ), with a more visible dysfunction when using ultrasounds vs. plethysmography (post-hoc comparison using Bonferroni correction, $P<0.01$ ). 


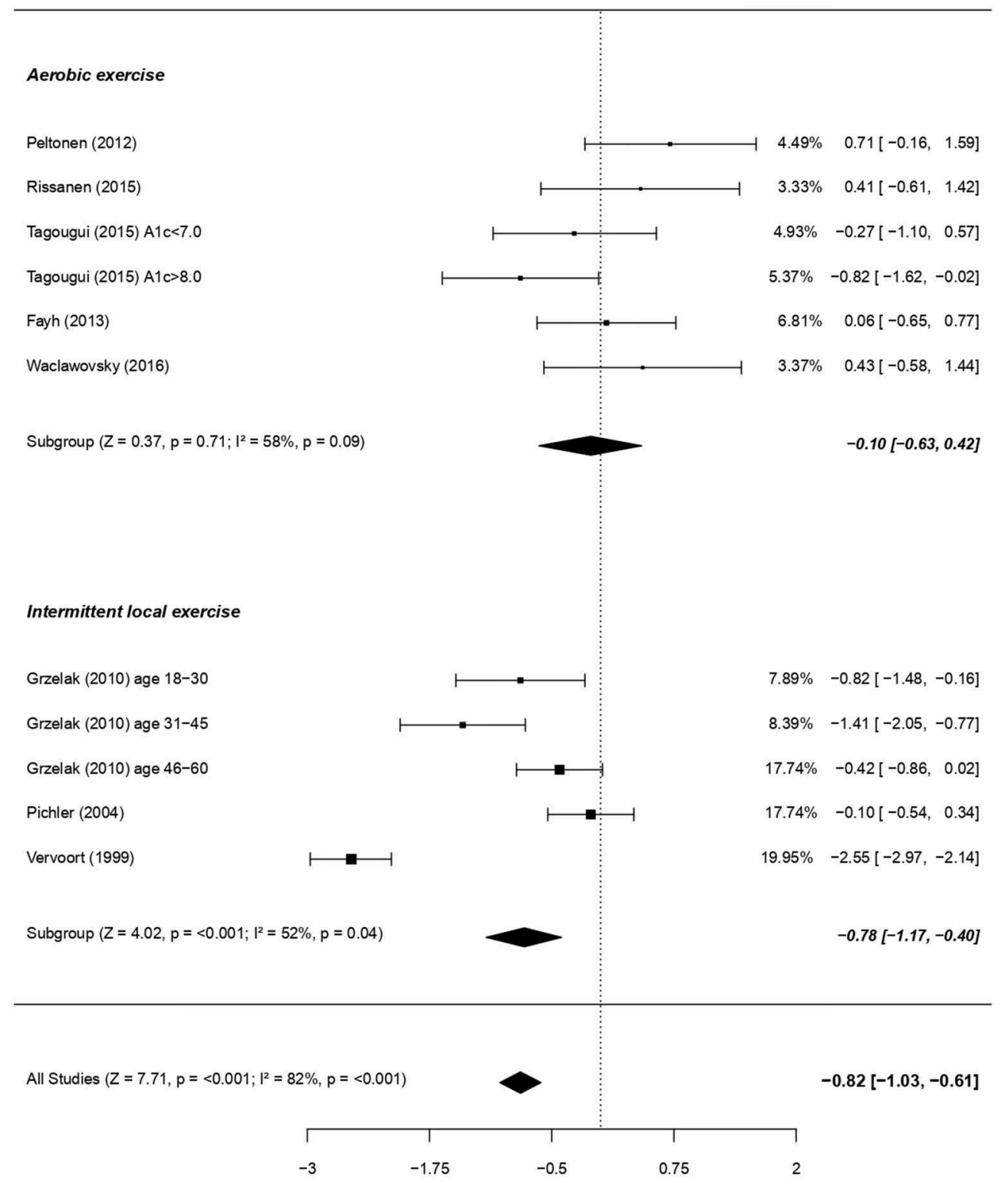

FIGURE 5 | Forest plots of studies on endothelial function in response to exercise, according to the type of exercise, in subjects with type 1 diabetes vs. controls. For each subgroup, the following values are indicated: $Z$ and $p$-value; $l^{2}$, heterogeneity and $p$ for heterogeneity. When comparing the two types of exercise with metaregressions (for qualitative data), the difference was significant (moderator $P=0.04$ ), with a more visible dysfunction when using intermittent local exercise vs.

aerobic exercise.

\section{DISCUSSION}

This meta-analysis, including 58 papers, attests to a medium-tolarge impairment of endothelial function in patients with type 1 diabetes free from clinically relevant vascular complications. This novel result is crucial considering that $\sim 30 \%$ of the studies on this topic failed to specifically detect this endothelial dysfunction (Table 1). Alterations of VSM function in type 1 diabetes are even less well-documented (only 8 of 21 studies attested to a significant difference; Table 1), but our current meta-analysis clearly demonstrates, for the first time, that aberrations in endothelial function is accompanied by a small-to-medium (according to Cohen's effects sizes) significant VSM dysfunction.

This impairment in VSM is alarming, considering that this dysfunction might be an even better predictor of atherosclerosis risk than endothelial dysfunction (74). Indeed, a significant negative correlation of NMD, but not FMD, with aortic intimamedia thickness, a sensitive marker of atherosclerosis, has been reported in 406 adolescents with type 1 diabetes (42). In line with 


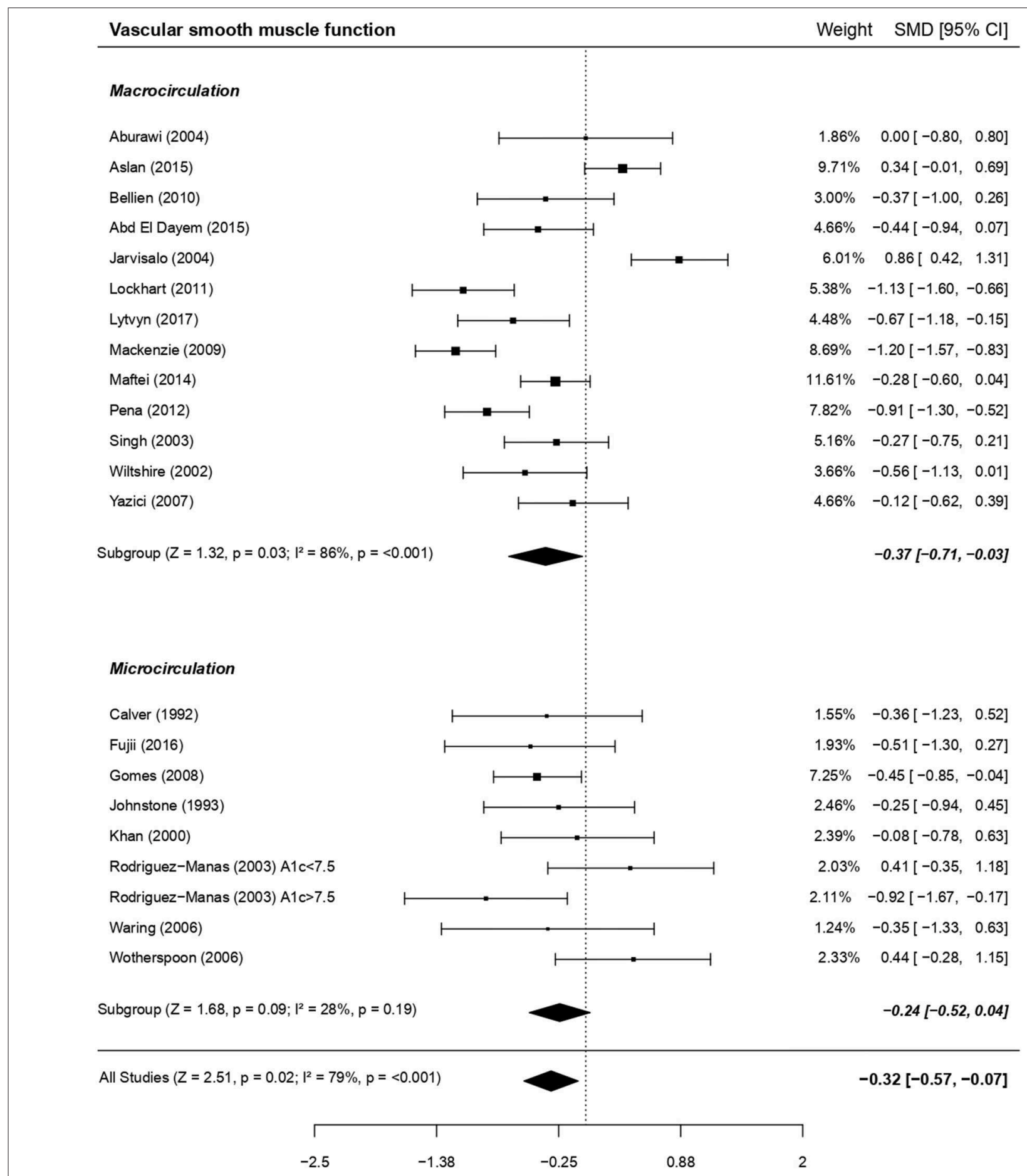

FIGURE 6 | Forest plot of studies on VSM function in subjects with type 1 diabetes vs. controls. For each subgroup, the following values are indicated: $Z$ and $p$-value; $R^{2}$, heterogeneity and $p$ for heterogeneity. There was no difference between macro and microcirculation (with metaregression for qualitative data: moderator $P=0.60$ ).

this notion, we found that a poorer VSM function was associated with higher triglycerides and, when focusing on macrovessels, with higher SBP and DBP. The link between VSM and these classical cardiovascular risk factors has been previously reported in type 2 diabetes (75), and may thus represent a common feature of vascular dysfunction in metabolic disease. In contrast to type 2 diabetes (75), low HDL-C did not appear to worsen VSM dysfunction in the current study. This is in line with the observation that HDL-C levels are elevated rather than decreased in type 1 diabetes (76), albeit with an alteration of 
their composition and function (77). Moreover, contrary to TG, HDL-C levels were described as poor predictors of micro- and macro-vascular complications in type 1 diabetes (78).

In addition to the smooth muscle dysfunction unmasked in the present meta-analysis, endothelial function tended to be still more markedly impaired. The latter result needs nevertheless cautious consideration as its assessment includes both the capacity of the endothelium to release vasodilator substances and the reactivity of the vascular smooth muscle. By the way, Khan et al. (36) revealed a correlation between iontophoresis Ach- and SNP-induced vasodilatation among children and adolescents with uncomplicated type 1 diabetes (36). Endothelial dysfunction was detected in both micro- and macro-vascular beds, albeit to a greater degree in the latter. Impaired endothelial dysfunction in macro- vs. micro-circulation in type 1 diabetes has to be considered in the context of the relative contribution of $\mathrm{NO}$ to the overall endotheliumdependent vasodilatation, which varies with vessels size. While $\mathrm{NO}$ appears crucial for vasodilatation in relatively large arteries and arterioles (79), endothelial derived hyperpolarizing factor (EDHF) makes a larger contribution to endothelial function in resistance arteries (i.e., the microcirculation) (80). Conceivably, EDHF may compensate deficiencies in NO bioactivity, as indicated in a rat model of type 1 diabetes (81). Likewise, as demonstrated in the forearm microcirculation of humans with type 1 diabetes using a cyclo-oxygenase inhibitor, prostanoidmediated vasodilatation may compensate for a lack of NO (82). Finally, we cannot exclude that additional alterations occur in muscular vasoconstrictive capacity within arterioles, as hypothesized in a study in children and adolescents with type 1 diabetes where capillary peak perfusion during reactive hyperemia was even increased in patients vs. controls (53). In any case, our novel finding that subtle endothelial dysfunction affects more large than small vessels was unexpected, reinvigorating the debate as to whether or not microvascular complications precede macrovascular complications (83).

From a clinical perspective, it is important to note that $\mathrm{HbA}_{1 \mathrm{c}}$, the traditional diabetes monitoring tool, was the only one risk factor holding a significant deleterious impact on endothelial function, especially in young patients. Future studies will nevertheless be needed to confirm the conditions of this impact since we were able to evidence it only when all studies were included, but not in the adult subgroup or separately in micro and macro-vessels. Earlier studies already demonstrated a negative correlation between high $\mathrm{HbA}_{1 \mathrm{c}}$ and acetylcholineinduced vasodilation in children, adolescents and young adults with type 1 diabetes $(36,84)$ or heat-induced microvascular dilation in adolescents and young adults with type 1 diabetes (85). In one of our previous studies, we split the group with type 1 diabetes into two subgroups, with either adequate $(<7 \%)$ or poor $(>8 \%)$ glycemic control and demonstrated an altered exercise-induced microvascular reactivity only in the poorlycontrolled group (57). Likewise, Hoffman et al. (86) revealed a more impaired reactive hyperaemic response in the human forearm of adolescents with $\mathrm{HbA}_{1 \mathrm{c}}>8.3 \%$.

The close link between $\mathrm{HbA}_{1 \mathrm{c}}$ levels and endothelial function may originate from one of two processes (i) the indirect deleterious impact of chronic hyperglycemia (as reflected by high $\mathrm{HbA}_{1 \mathrm{c}}$ levels) on NO bioavailability through reactive oxygen species overproduction, which inactivate $\mathrm{NO}$ by conversion into peroxynitrite and promote ADMA production and arginase overexpression, thus inhibiting eNOS activity and reducing the availability of the substrate L-arginine (87), (ii) a possible direct impact of glycated hemoglobin, whose affinity for NO is greater than that of the non-glycated molecule (88). This implies that, among patients with high $\mathrm{HbA}_{1 \mathrm{c}}$ levels, $\mathrm{NO}$ transported (bound on $\mathrm{Hb}$ ) from regions of high production (i.e., the conduit arteries) may be less readily released downstream in the microcirculation, potentially altering microvascular tone.

Noteworthy, we did not detect any moderating effect of diabetes duration on endothelial dysfunction and we found that the adverse impact of $\mathrm{HbA}_{1 \mathrm{c}}$ was evident already in childhood and adolescence, which highlights that optimization of glycemic control should be at the center of care from the earliest stages of life onward.

Our meta-analysis did not reveal any negative influence of lipid profile or BMI on the association between endothelial dysfunction and type 1 diabetes. This result is not too surprising considering that most study participants displayed mean lipid profiles and BMI within ranges of normality (except for 1 of the 35 studies on HDL-C, 1 among the 47 studies on cholesterol). Accordingly, the majority of studies that tested the association between lipid profile and endothelial function in uncomplicated type 1 diabetes did not reveal any link between these outcomes $(14,16,19,45,54,56)$.

Considering the high heterogeneity in the results, we have also separated the analyses of endothelial function according to the size of vessels studied and the type of stimulus/intervention used. Separately, the effects of some stimuli (i.e., exercise and pharmacological substances) did not reach statistical significance only for small vessels. For large vessels, FMD and exercise stimuli were both associated with a significant endothelial dysfunction among the patients. In addition, when comparing the effects of the stimuli between each other, in macro as well as in microvessels, no significant differences appeared. This suggests that physical exercise, a physiological stimulus, may be efficient in triggering endothelial NO release, particularly in large vessels. In a previous study, Grzelak et al. (31) demonstrated that exercise (i.e., handgrip) was even more efficient than occlusionreperfusion maneuver for promoting a dilatation of the artery in adults with type 1 diabetes. Only 10 studies have investigated the impact of an acute exercise stimulus on vascular function in uncomplicated patients with type 1 diabetes compared to a healthy population; 3 of these focused on skin capillaries $(12,28,59), 7$ on muscle microvasculature $(26,47,49,51,57$, $60)$ and 3 on arteries (31). When analyzing more specifically the type of exercise chosen, only one work used a session of resistance exercise and compared it to aerobic exercise of the same duration, without finding any intergroup difference of post-exercise forearm blood flow, regardless of the type of exercise performed (60). Intriguingly, concentric intermittent local (handgrip) exercise [4 cohorts; $(31,49)$ ] appeared to induce a significantly greater vascular impairment in patients with type 1 diabetes than when performing whole-body aerobic 
exercise [ 6 cohorts; $(26,47,51,57,60)]$. Whether this interexercise difference results from a release of different and exercisespecific vasoactive molecules remains to be elucidated. Of note, isometric local exercise involves a very small active muscle mass, where blood flow is unlikely to be influenced by variations in cardiac output (89). Conversely, whole body aerobic exercise elicits substantial increases in cardiac output, and this central cardiovascular response might mask inter-group differences in peripheral vascular reactivity. Noteworthy, focusing only on local intermittent exercise allowed to considerably reduce heterogeneity to a level close to the "moderate" heterogeneity category. Finally, the ability of local isometric intermittent exercise to detect endothelial dysfunction in uncomplicated patients with type 1 diabetes provides considerable clinical perspective: such an exercise, which is physiological, cheap (requiring only a handgrip) and simple to implement, would be worth adding to the routine clinical follow-ups of uncomplicated patients, in whom pharmacological or painful (e.g., $5 \mathrm{~min}$ occlusion) stimuli are less well-tolerated.

\section{Heterogeneity}

In this meta-analysis, the heterogeneity was very high in practically all the analyzed topics.

The large number of studies in this meta-analysis revealed considerable variability in methodological practice which is likely to contribute to the heterogeneity in responses observed. Factors such as the inclusion criteria and precautions before the visit or the $\mathrm{D}$-day, matching between patients with type 1 diabetes and healthy controls, varied widely between studies (Table 1), inviting bias for meta-analyses. Most of the studies did match their populations only for age. Focusing on the 10 studies on exercise, only 3 indicated a matching for physical activity level. Smoking, oral contraceptives or statins could have an impact on vasoreactivity, but unfortunately this is not always considered. In addition, prevailing circulating glucose and insulin concentrations, which presumably differ considerably between subjects and studies but are regrettably not always reported (of the 58 studies, only 31 displayed glycemia, 23 reported the status of insulin injection among which 17 were in fasting and 16 in a post-prandial state), are known as modulating peripheral vasodilation $(90,91)$. Ultimately, while the influence of long-term glycemic control (i.e., $\mathrm{HbA}_{1 \mathrm{c}}$ ) on vascular function is systematically taken into consideration, only 2 studies (14, 48), among the 58 included in the meta-analysis, explored the moderating effect of glycemic variability (i.e., oscillating glucose concentrations) or hypoglycaemia on vascular function in patients with type 1 diabetes compared to healthy controls. These 2 studies, in children with type 1 diabetes, failed to detect significant correlation between FMD and glycemic variability, as assessed using 2-week, seven-point, self-monitored blood glucose logs (14) or 48-h continuous glucose monitoring (48). However, as previous in vitro (92) and in vivo (21,93-98), studies suggested a possible deleterious impact of glycemic variability on vascular function, further studies are needed to better explore the strength, conditions and mechanisms of this impact. An increased magnitude of glycemic variability would generate more reactive oxygen species (including nitrotyrosine) in complications-prone cells compared to stable hyperglycemia and preliminary data suggest that protective adaptations induced by constant exposure to hyperglycemia are inadequately activated with intermittent exposure, allowing for more pronounced toxicity (92). Besides, in accordance with other reports $(96,97)$, Pena et al. (48) showed that an index of hypoglycaemic risk (i.e., Glycemic Risk Assessment Diabetes Equation-Hypoglycemia), measured over the 48 preceding hours, was a negative predictor of FMD but not of NMD. Noteworthy, hypoglycemia may induce vascular damages in the short and long terms: the hypoglycaemia-induced acute hemodynamic changes may precipitate and aggravate a vascular event during an acute episode (99), while repetition of hypoglycemic events could trigger abnormalities of coagulation, fibrinolysis, and inflammation.

Likewise, the combination of various ages, genders, regions assessed and, mainly, methods of assessing vascular function may greatly contribute to the high heterogeneity. In that respect, we performed metaregressions and separated sub-analyses of each intervention. Although some parameters partly explained some heterogeneity ( $c f$., significant moderator $P$-values), the heterogeneity remained high in all the sub-analyses, thus limiting the extrapolation of the effect size (73). This result highlights the urgent need for vascular methods standardization. Although the gold-standard ultrasonographic assessment of large vessels by FMD is the only method benefiting from standardized guidelines $(100,101)$, it is operator-dependant and demands considerable practice before reproducible results are obtained $(102,103)$. In addition, very few studies (i.e., 1 among 30 included in the meta-analysis) took into account evoked hyperaemic shear stress while analysing FMD, while this, if altered, can constitute a reduced stimulus for dilation (104). While efforts for microvascular assessment standardization are emerging (105), further work in this direction is needed. As in this current metaanalysis, a particular technique of measure is typically appointed to correspond to either macro- or micro-vascular circulation, but in real-life these cardiovascular functions are, of course, interconnected and interdependent.

\section{Limits and Perspectives}

While this meta-analysis focused only on peripheral vessels, vascular dysfunction is not limited to this area. Vascular dysfunction may also appear in cerebral vessels in response to aerobic exercise (106), which is highly relevant given the risk for long-term diabetes-associated cognitive decline (107).

Even if endothelial and, to a lesser extent, VSM dysfunction, have been the topic of a large number of studies in uncomplicated patients with type 1 diabetes, further studies focusing on the understanding of their underlying molecular mechanisms would benefit from agreeing on the most sensitive stimulus and most appropriate vascular bed to study for relevant routine clinical patient follow-up. NO is the main mediator controlling vascular tone and any reduction in its bioavailibility (e.g., by superoxide) translates directly into endothelial dysfunction. However, even the NO receptor (soluble guanylyl cyclase; sGC) is sensitive to oxidative stress, and a redox-driven impairment of $\mathrm{NO} / \mathrm{sGC}$ signaling may contribute to VSM dysfunction. Moreover, many others pathways are involved in vasodilatation 
and each stimulus induces vasodilatation through common but highly specific pathways [e.g., prostaglandin (108) and EDHF/EET (epoxyeicosatrienoic acids) pathway in response to post-occlusive reactive hyperemia, chemical factors released by contracting skeletal muscle (109)].

Up to now, very limited data is available about underlying mechanisms of vasodilation defects in uncomplicated type 1 diabetes. Of the 55 studies included in this meta-analysis, only 4 attempted to concomitantly assess some of the putative underlying mechanisms of the vasoreactivity defect. Although Fayh et al. (26) hypothesized, by measuring total NOx (the sum of nitrite and nitrate), that NO production was unaltered in their study, this result merits confirmation because the authors did not distinguish nitrite and nitrate, although only nitrite reflects acute changes in NO synthase activity in humans (110). Using pharmacological inhibitors of endogenous NO synthesis (L-NMMA) and prostaglandinmediated vasodilatation (indomethacin) (63) or of $\mathrm{Ca}^{2+}$ release in cytoplasm (35), administered concomitantly with vasoactive substances [acetylcholine or exogenous NO (sodium nitroprusside), respectively], no differences were found between patients with type 1 diabetes and healthy controls suggesting normal contribution of $\mathrm{NO}$ and prostaglandin as well as efficient VSM calcium channels. However, Rodriguez-Manas et al. (52) suggested the involvement of oxidative stress in endothelial dysfunction among poorly-controlled type 1 diabetes subjects: they indeed demonstrated an improvement of blunted vasodilatory response to metacholine when co-infusing superoxide dismutase in patients with $\mathrm{HbA}_{1 \mathrm{c}} \geq 7.5 \%$, while no changes appeared for healthy controls or well-controlled patients.

In addition to help selecting the most appropriate stimulus for routine follow-ups of vascular function in type 1 diabetes, future efforts should focus on a better understanding of the underlying mechanisms to design specific interventions and molecular target to slow down progressive vascular damage. Several studies already tested some non-pharmacological interventions in patients with uncomplicated type 1 diabetes and displayed encouraging results for regular physical exercise (111) and nutritional supplementation with L-arginine (26) and vitamin C (112).

In conclusion, this meta-analysis not only corroborates the presence of a medium-to-large impairment of endothelial function, even in response to physiological stimuli such as exercise, but also highlights a VSM dysfunction in children

\section{REFERENCES}

1. Nathan DM, Cleary PA, Backlund JY, Genuth SM, Lachin JM, Orchard TJ, et al. Intensive diabetes treatment and cardiovascular disease in patients with type 1 diabetes. $N$ Engl J Med. (2005) 353:2643-53. doi: 10.1056/NEJMoa052187

2. Jarvisalo MJ, Raitakari M, Toikka JO, Putto-Laurila A, Rontu $\mathrm{R}$, Laine $\mathrm{S}$, et al. Endothelial dysfunction and increased arterial intima-media thickness in children with type 1 diabetes. Circulation. (2004) 109:1750-5. doi: 10.1161/01.CIR.0000124725.46 165.2C and adults with type 1 diabetes free from clinical vascular complications. Of note, heterogeneity was high and was not further explained by subgroups analyses, thus limiting the extrapolation of the effect size and highlighting the urgent need for vascular methods standardization. Surprisingly, endothelial dysfunction seemed more marked in large rather than small blood vessels, re-invigorating the debate about the timing and complexity of the development of vascular complications in type 1 diabetes. The inverse relationship between endothelial function and $\mathrm{HbA}_{1 \mathrm{c}}$ provides further arguments for identifying therapeutic strategies aimed at improving glycemic control.

\section{DATA AVAILABILITY STATEMENT}

All datasets generated for this study are included in the article/Supplementary Material.

\section{AUTHOR CONTRIBUTIONS}

EL and EH extracted and analyzed data and wrote the manuscript. LD performed statistical analyses. PF gave his clinical expertise to the inclusion of studies in the metaanalysis and resolved discrepancies by consensus in the case of disagreements on eligibility criteria between EL and EH. PF, LD, $\mathrm{CB}, \mathrm{MF}, \mathrm{JB}, \mathrm{SB}, \mathrm{MP}-\mathrm{C}$, and MR were involved in interpreting data and reviewing the manuscript. $\mathrm{EH}$ is the guarantor of this work and, as such, had full access to all data and takes responsibility for the integrity of the data and the accuracy of the data analysis.

\section{ACKNOWLEDGMENTS}

We thank all those authors of the primary articles who responded to our queries. We also wish to thank Dr. Cajsa Tonoli for her help at the beginning of this meta-analysis. We thank Pierre Morel for his help with preparing the figures. EL is recruited as a post-doctoral researcher thanks to a donation from Linde Homecare France.

\section{SUPPLEMENTARY MATERIAL}

The Supplementary Material for this article can be found online at: https://www.frontiersin.org/articles/10.3389/fendo. 2020.00203/full\#supplementary-material
3. Mahmud FH, Earing MG, Lee RA, Lteif AN, Driscoll DJ, Lerman A. Altered endothelial function in asymptomatic male adolescents with type 1 diabetes. Congenit Heart Dis. (2006) 1:98-103. doi: 10.1111/j.1747-0803.2006.00015.x

4. Wink DA, Miranda KM, Espey MG, Pluta RM, Hewett SJ, Colton C, et al. Mechanisms of the antioxidant effects of nitric oxide. Antioxid Redox Signal. (2001) 3:203-13. doi: 10.1089/152308601300185179

5. Quyyumi AA. Endothelial function in health and disease: new insights into the genesis of cardiovascular disease. Am J Med. (1998). 105:32S-9S. doi: 10.1016/S0002-9343(98)00209-5

6. Bugiardini R. Endothelial function and the prediction of CVD in diabetes. Curr Diabetes Rep. (2006) 6:17-21. doi: 10.1007/s11892-006-0046-3 
7. Ce GV, Rohde LE, da Silva AM, Punales MK, de Castro AC, Bertoluci MC. Endothelial dysfunction is related to poor glycemic control in adolescents with type 1 diabetes under 5 years of disease: evidence of metabolic memory. J Clin Endocrinol Metab. (2011) 96:1493-9. doi: 10.1210/jc. 2010-2363

8. Son SM, Whalin MK, Harrison DG, Taylor WR, Griendling KK. Oxidative stress and diabetic vascular complications. Curr Diabetes Rep. (2004) 4:24752. doi: 10.1007/s11892-004-0075-8

9. Abd El Dayem SM, El Magd El Bohy A, Battah AA. Carotid intimal medial thickness and its relation to endothelial dysfunction and echocardiographic changes in adolescents with type 1 diabetes. J Pediatr Endocrinol Metab. (2015) 28:1029-37. doi: 10.1515/jpem-2014-0355

10. Abi-Chahin TC, Hausen Mde A, Mansano-Marques CM, Halfoun VL. Microvascular reactivity in type 1 diabetics. Arq Bras Endocrinol Metabol. (2009) 53:741-6. doi: 10.1590/S0004-27302009000 600008

11. Aburawi E, Liuba P, Pesonen E, Yla-Herttuala S, Sjoblad S. Acute respiratory viral infections aggravate arterial endothelial dysfunction in children with type 1 diabetes. Diabetes Care. (2004) 27:27335. doi: 10.2337/diacare.27.11.2733

12. Allen JD, Welsch M, Aucoin N, Wood R, Lee M, LeBlanc KE. Forearm vasoreactivity in type 1diabetic subjects. Can J Appl Physiol. (2001) 26:3443. doi: 10.1139/h01-003

13. Aslan AN, Keles T, Ayhan H, Kasapkara HA, Akcay M, Durmaz T, et al. The relationship between epicardial fat thickness and endothelial dysfunction in type I diabetes mellitus. Echocardiography. (2015) 32:174553. doi: 10.1111/echo.12960

14. Babar GS, Zidan H, Widlansky ME, Das E, Hoffmann RG, Daoud $\mathrm{M}$, et al. Impaired endothelial function in preadolescent children with type 1 diabetes. Diabetes Care. (2011) 34:681-5. doi: 10.2337/dc $10-2134$

15. Bayir O, Korkmaz HA, Dizdarer C, Mese T, Tavli V. Carotid artery intima-media thickness in pediatric type 1 diabetic patients. Anadolu Kardiyol Derg. (2014) 14:464-70. doi: 10.5152/akd. 2013.4788

16. Bellien J, Costentin A, Dutheil-Maillochaud B, Iacob M, Kuhn JM, Thuillez C, et al. Early stage detection of conduit artery endothelial dysfunction in patients with type 1 diabetes. Diabetes Vasc Dis Res. (2010) 7:15866. doi: 10.1177/1479164109360470

17. Boolell M, Tooke JE. The skin hyperaemic response to local injection of substance P and capsaicin in diabetes mellitus. Diabet Med. (1990) 7:898901. doi: 10.1111/j.1464-5491.1990.tb01325.x

18. Bradley TJ, Slorach C, Mahmud FH, Dunger DB, Deanfield J, Deda L, et al. Early changes in cardiovascular structure and function in adolescents with type 1 diabetes. Cardiovasc Diabetol. (2016) 15:31. doi: 10.1186/s12933-016-0351-3

19. Bruzzi P, Predieri B, Patianna VD, Salvini A, Rossi R, Modena MG, et al. Longitudinal evaluation of endothelial function in children and adolescents with type 1 diabetes mellitus: a long-term follow-up study. Pediatr Int. (2014) 56:188-95. doi: 10.1111/ped.12220

20. Calver A, Collier J, Vallance P. Inhibition and stimulation of nitric oxide synthesis in the human forearm arterial bed of patients with insulindependent diabetes. J Clin Invest. (1992) 90:2548-54. doi: 10.1172/JCI 116149

21. Ceriello A, Novials A, Ortega E, La Sala L, Pujadas G, Testa R, et al. Evidence that hyperglycemia after recovery from hypoglycemia worsens endothelial function and increases oxidative stress and inflammation in healthy control subjects and subjects with type 1 diabetes. Diabetes. (2012) 61:2993-7. doi: 10.2337/db12-0224

22. Chiesa ST, Charakida M, McLoughlin E, Nguyen HC, Georgiopoulos G, Motran L, et al. Elevated high-density lipoprotein in adolescents with Type 1 diabetes is associated with endothelial dysfunction in the presence of systemic inflammation. Eur Heart J. (2019). doi: 10.1093/eurheartj/ ehz114

23. Ciftel M, Ertug H, Parlak M, Akcurin G, Kardelen F. Investigation of endothelial dysfunction and arterial stiffness in children with type 1 diabetes mellitus and the association with diastolic dysfunction. Diabetes Vasc Dis Res. (2014) 11:19-25. doi: 10.1177/1479164113508564
24. DiMeglio LA, Tosh A, Saha C, Estes M, Mund J, Mead LE, et al. Endothelial abnormalities in adolescents with type 1 diabetes: a biomarker for vascular sequelae? J Pediatr. (2010) 157:540-6. doi: 10.1016/j.jpeds.2010. 04.050

25. Eltayeb AA, Ahmad FA, Sayed DM, Osama AM. Subclinical vascular endothelial dysfunctions and myocardial changes with type 1 diabetes mellitus in children and adolescents. Pediatr Cardiol. (2014) 35:96574. doi: 10.1007/s00246-014-0883-9

26. Fayh AP, Krause M, Rodrigues-Krause J, Ribeiro JL, Ribeiro JP, Friedman $\mathrm{R}$, et al. Effects of L-arginine supplementation on blood flow, oxidative stress status and exercise responses in young adults with uncomplicated type I diabetes. Eur J Nutr. (2013) 52:975-83. doi: 10.1007/s00394012-0404-7

27. Franzeck UK, Stengele B, Panradl U, Wahl P, Tillmanns H. Cutaneous reactive hyperemia in short-term and long-term type I diabetes-continuous monitoring by a combined laser Doppler and transcutaneous oxygen probe. Vasa. (1990) 19:8-15.

28. Fujii N, Dervis S, Sigal RJ, Kenny GP. Type 1 diabetes modulates cyclooxygenase- and nitric oxide-dependent mechanisms governing sweating but not cutaneous vasodilation during exercise in the heat. Am J Physiol Regul Integr Comp Physiol. (2016) 311:R1076-R84. doi: 10.1152/ajpregu.00376.2016

29. Glowinska-Olszewska B, Moniuszko M, Hryniewicz A, Jeznach M, Rusak M, Dabrowska $M$, et al. Relationship between circulating endothelial progenitor cells and endothelial dysfunction in children with type 1 diabetes: a novel paradigm of early atherosclerosis in high-risk young patients. Eur J Endocrinol. (2013) 168:153-61. doi: 10.1530/EJE12-0857

30. Gomes MB, Matheus AS, Tibirica E. Evaluation of microvascular endothelial function in patients with type 1 diabetes using laser-Doppler perfusion monitoring: which method to choose? Microvasc Res. (2008) 76:1323. doi: 10.1016/j.mvr.2008.04.003

31. Grzelak P, Olszycki M, Majos A, Czupryniak L, Strzelczyk J, Stefanczyk L. Hand exercise test for the assessment of endothelium-dependent vasodilatation in subjects with type 1 diabetes. Diabetes Technol Ther. (2010) 12:605-11. doi: 10.1089/dia.2010.0001

32. Haak E, Haak T, Kusterer K, Reschke B, Faust H, Usadel KH. Microcirculation in hyperglycemic patients with IDDM without diabetic complications-effect of low-dose angiotensinconverting enzyme inhibition. Exp Clin Endocrinol Diabetes. (1998) 106:45-50. doi: 10.1055/s-0029-1211949

33. Heier M, Espeland CN, Brunborg C, Seljeflot I, Margeirsdottir HD, Hanssen KF, et al. Preserved endothelial function in young adults with type 1 diabetes. PLoS One. (2018) 13:e0206523. doi: 10.1371/journal.pone. 0206523

34. Hoffman RP, Copenhaver MM, Zhou D, Yu CY. Increased body fat and reduced insulin sensitivity are associated with impaired endothelial function and subendocardial viability in healthy, non-Hispanic white adolescents. Pediatr Diabetes. (2019) 20:842-8. doi: 10.1111/pedi.12896

35. Johnstone MT, Creager SJ, Scales KM, Cusco JA, Lee BK, Creager MA. Impaired endothelium-dependent vasodilation in patients with insulin-dependent diabetes mellitus. Circulation. (1993) 88:2510-6. doi: 10.1161/01.CIR.88.6.2510

36. Khan F, Elhadd TA, Greene SA, Belch JJ. Impaired skin microvascular function in children, adolescents, and young adults with type 1 diabetes. Diabetes Care. (2000) 23:215-20. doi: 10.2337/diacare.23.2.215

37. Koitka A, Abraham P, Bouhanick B, Sigaudo-Roussel D, Demiot C, Saumet JL. Impaired pressure-induced vasodilation at the foot in young adults with type 1 diabetes. Diabetes. (2004) 53:721-5. doi: 10.2337/diabetes. 53.3.721

38. Lockhart CJ, Agnew CE, McCann A, Hamilton PK, Quinn CE, McCall DO, et al. Impaired flow-mediated dilatation response in uncomplicated Type 1 diabetes mellitus: influence of shear stress and microvascular reactivity. Clin Sci (Lond). (2011) 121:129-39. doi: 10.1042/CS20100448

39. Lytvyn Y, Mahmud FH, Daneman D, Deda L, Dunger DB, Deanfield J, et al. Association between plasma uric acid levels and cardiorenal function in adolescents with type 1 diabetes. Diabetes Care. (2016) 39:6116. doi: $10.2337 / \mathrm{dc} 15-2345$ 
40. Lytvyn Y, Har R, Locke A, Lai V, Fong D, Advani A, et al. Renal and vascular effects of uric acid lowering in normouricemic patients with uncomplicated type 1 diabetes. Diabetes. (2017) 66:1939-49. doi: 10.2337/db $17-0168$

41. MacKenzie KE, Wiltshire EJ, Pena AS, Gent R, Hirte C, Piotto L, et al. HsCRP is associated with weight, BMI, and female sex but not with endothelial function in children with type 1 diabetes. Pediatr Diabetes. (2009) 10:4451. doi: 10.1111/j.1399-5448.2008.00456.x

42. Maftei O, Pena AS, Sullivan T, Jones TW, Donaghue KC, Cameron FJ, et al. Early atherosclerosis relates to urinary albumin excretion and cardiovascular risk factors in adolescents with type 1 diabetes: adolescent type 1 diabetes cardio-renal intervention trial (AdDIT). Diabetes Care. (2014) 37:306975. doi: $10.2337 / \mathrm{dc} 14-0700$

43. Mahmud FH, Van Uum S, Kanji N, Thiessen-Philbrook H, Clarson CL. Impaired endothelial function in adolescents with type 1 diabetes mellitus. J Pediatr. (2008) 152:557-62. doi: 10.1016/j.jpeds.2007. 08.044

44. Nascimento A, Sequeira IJ, Vasconcelos DF, Gandolfi L, Pratesi R, Nobrega YKM. Endothelial dysfunction in children with type 1 diabetes mellitus. Arch Endocrinol Metab. (2017) 61:476-83. doi: 10.1590/2359-39970000 00271

45. Palombo C, Kozakova M, Morizzo C, Gnesi L, Barsotti MC, Spontoni P, et al. Circulating endothelial progenitor cells and large artery structure and function in young subjects with uncomplicated type 1 diabetes. Cardiovasc Diabetol. (2011) 10:88. doi: 10.1186/1475-2840-10-88

46. Pareyn A, Allegaert K, Asscherickx W, Peirsman E, Verhamme P, Casteels $\mathrm{K}$. Impaired endothelial function in female adolescents with type 1 diabetes measured by peripheral artery tonometry. Eur J Pediatr. (2013) 172:101722. doi: 10.1007/s00431-013-1988-5

47. Peltonen JE, Koponen AS, Pullinen K, Hagglund H, Aho JM, Kyrolainen $\mathrm{H}$, et al. Alveolar gas exchange and tissue deoxygenation during exercise in type 1 diabetes patients and healthy controls. Respir Physiol Neurobiol. (2012) 181:267-76. doi: 10.1016/j.resp.2012.04.002

48. Pena AS, Couper JJ, Harrington J, Gent R, Fairchild J, Tham E, et al. Hypoglycemia, but not glucose variability, relates to vascular function in children with type 1 diabetes. Diabetes Technol Ther. (2012) 14:45762. doi: 10.1089/dia.2011.0229

49. Pichler G, Urlesberger B, Jirak P, Zotter H, Reiterer E, Muller W, et al. Reduced forearm blood flow in children and adolescents with type 1 diabetes (measured by near-infrared spectroscopy). Diabetes Care. (2004) 27:19426. doi: 10.2337/diacare.27.8.1942

50. Pillay S, Anderson J, Couper J, Maftei O, Gent R, Pena AS. Children with type 1 diabetes have delayed flow-mediated dilation. Can J Diabetes. (2018) 42:276-80. doi: 10.1016/j.jcjd.2017.06.011

51. Rissanen AP, Tikkanen HO, Koponen AS, Aho JM, Peltonen JE. Central and peripheral cardiovascular impairments limit $\mathrm{VO}(2$ peak) in type 1 diabetes. Med Sci Sports Exerc. (2015) 47:223-30. doi: 10.1249/MSS.0000000000000419

52. Rodriguez-Manas L, Lopez-Doriga P, Petidier R, Neira M, Solis J, Pavon I, et al. Effect of glycaemic control on the vascular nitric oxide system in patients with type 1 diabetes. J Hypertens. (2003) 21:113743. doi: 10.1097/00004872-200306000-00013

53. Schlager O, Hammer A, Willfort-Ehringer A, Fritsch M, Rami-Merhar B, Schober E, et al. Microvascular autoregulation in children and adolescents with type 1 diabetes mellitus. Diabetologia. (2012) 55:163340. doi: $10.1007 / \mathrm{s} 00125-012-2502-8$

54. Singh TP, Groehn H, Kazmers A. Vascular function and carotid intimal-medial thickness in children with insulin-dependent diabetes mellitus. J Am Coll Cardiol. (2003) 41:661-5. doi: 10.1016/S0735-1097(02) 02894-2

55. Sochett E, Noone D, Grattan M, Slorach C, Moineddin R, Elia Y, et al. Relationship between serum inflammatory markers and vascular function in a cohort of adolescents with type 1 diabetes. Cytokine. (2017) 99:2339. doi: 10.1016/j.cyto.2017.07.013

56. Tacito LHB, Pires AC, Yugar-Toledo JC. Impaired flow-mediated dilation response and carotid intima-media thickness in patients with type 1 diabetes mellitus with a mean disease duration of 4.1 years. Arch Endocrinol Metab. (2017) 61:542-9. doi: 10.1590/2359-3997000000281
57. Tagougui S, Leclair E, Fontaine P, Matran R, Marais G, Aucouturier J, et al. Muscle oxygen supply impairment during exercise in poorly controlled type 1 diabetes. Med Sci Sports Exerc. (2015) 47:2319. doi: 10.1249/MSS.0000000000000424

58. Tibirica E, Rodrigues E, Cobas RA, Gomes MB. Endothelial function in patients with type 1 diabetes evaluated by skin capillary recruitment. Microvasc Res. (2007) 73:107-12. doi: 10.1016/j.mvr.2006. 11.004

59. Vervoort G, Lutterman JA, Smits P, Berden JH, Wetzels JF. Transcapillary escape rate of albumin is increased and related to haemodynamic changes in normo-albuminuric type 1 diabetic patients. J Hypertens. (1999) $17(12 \mathrm{Pt}$ 2):1911-6. doi: 10.1097/00004872-199917121-00021

60. Waclawovsky G, Umpierre D, Figueira FR, De Lima ES, Alegretti AP, Schneider L, et al. Exercise on progenitor cells in healthy subjects and patients with type 1 diabetes. Med Sci Sports Exerc. (2016) 48:1909. doi: 10.1249/MSS.0000000000000764

61. Waring WS, McKnight JA, Webb DJ, Maxwell SR. Uric acid restores endothelial function in patients with type 1 diabetes and regular smokers. Diabetes. (2006) 55:3127-32. doi: 10.2337/db06-0283

62. Wiltshire EJ, Gent R, Hirte C, Pena A, Thomas DW, Couper JJ. Endothelial dysfunction relates to folate status in children and adolescents with type 1 diabetes. Diabetes. (2002) 51:2282-6. doi: 10.2337/diabetes.51. 7.2282

63. Wotherspoon F, Laight DW, Browne DL, Turner C, Meeking DR, Allard SE, et al. Plasma homocysteine, oxidative stress and endothelial function in patients with Type 1 diabetes mellitus and microalbuminuria. Diabetes Med. (2006) 23:1350-6. doi: 10.1111/j.1464-5491.2006.01980.x

64. Yazici D, Yavuz DG, Unsalan S, Toprak A, Yuksel M, Deyneli O, et al. Temporal effects of low-dose ACE inhibition on endothelial function in Type 1 diabetic patients. J Endocrinol Invest. (2007) 30:72633. doi: 10.1007/BF03350809

65. El-Najjar N, Kulkarni RP, Nader N, Hodeify R, Machaca K. Effects of hyperglycemia on vascular smooth muscle $\mathrm{Ca}^{2+}$ signaling. Biomed Res Int. (2017) 2017:3691349. doi: 10.1155/2017/3691349

66. Sun J, Xu Y, Dai Z, Sun Y. Intermittent high glucose enhances proliferation of vascular smooth muscle cells by upregulating osteopontin. Mol Cell Endocrinol. (2009) 313:64-9. doi: 10.1016/j.mce.2009.08.019

67. Wang P, Xu YY, Lv TT, Guan SY, Li XM, Li XP, et al. Subclinical atherosclerosis in patients with type 1 diabetes mellitus: a systematic review and meta-analysis. Angiology. (2019) 70:141-59. doi: 10.1177/0003319718787366

68. Fuchs D, Dupon PP, Schaap LA, Draijer R. The association between diabetes and dermal microvascular dysfunction non-invasively assessed by laser Doppler with local thermal hyperemia: a systematic review with meta-analysis. Cardiovasc Diabetol. (2017) 16:11. doi: 10.1186/s12933-0160487-1

69. Liberati A, Altman DG, Tetzlaff J, Mulrow C, Gotzsche PC, Ioannidis JP, et al. The PRISMA statement for reporting systematic reviews and meta-analyses of studies that evaluate health care interventions: explanation and elaboration. J Clin Epidemiol. (2009) 62:e1-34. doi: 10.1016/j.jclinepi.2009.06.006

70. Tibirica E, Rodrigues E, Cobas R, Gomes MB. Impairment of skin capillary recruitment precedes chronic complications in patients with type 1 diabetes. Rev Diabet Stud. (2007) 4:85-8. doi: 10.1900/RDS.2007.4.85

71. Vervoort G, Wetzels JF, Lutterman JA, van Doorn LG, Berden JH, Smits P. Elevated skeletal muscle blood flow in noncomplicated type 1 diabetes mellitus: role of nitric oxide and sympathetic tone. Hypertension. (1999) 34:1080-5. doi: 10.1161/01.HYP.34.5.1080

72. Wotherspoon F, Browne DL, Meeking DR, Allard SE, Munday LJ, Shaw $\mathrm{KM}$, et al. The contribution of nitric oxide and vasodilatory prostanoids to bradykinin-mediated vasodilation in Type 1 diabetes. Diabet Med. (2005) 22:697-702. doi: 10.1111/j.1464-5491.2005.01493.x

73. Melsen WG, Bootsma MC, Rovers MM, Bonten MJ. The effects of clinical and statistical heterogeneity on the predictive values of results from meta-analyses. Clin Microbiol Infect. (2014) 20:123-9. doi: 10.1111/1469-0691.12494

74. Yeboah J, Folsom AR, Burke GL, Johnson C, Polak JF, Post $\mathrm{W}$, et al. Predictive value of brachial flow-mediated dilation 
for incident cardiovascular events in a population-based study: the multi-ethnic study of atherosclerosis. Circulation. (2009) 120:502-9. doi: 10.1161/CIRCULATIONAHA.109.864801

75. Montero D, Walther G, Perez-Martin A, Vicente-Salar N, Roche E, Vinet A. Vascular smooth muscle function in type 2 diabetes mellitus: a systematic review and meta-analysis. Diabetologia. (2013) 56:212233. doi: 10.1007/s00125-013-2974-1

76. Colhoun HM, Taskinen MR, Otvos JD, Van Den Berg P, O'Connor J, Van Tol A. Relationship of phospholipid transfer protein activity to HDL and apolipoprotein B-containing lipoproteins in subjects with and without type 1 diabetes. Diabetes. (2002) 51:3300-5. doi: 10.2337/diabetes.51. 11.3300

77. Persegol L, Foissac M, Lagrost L, Athias A, Gambert P, Verges B, et al. HDL particles from type 1 diabetic patients are unable to reverse the inhibitory effect of oxidised LDL on endothelium-dependent vasorelaxation. Diabetologia. (2007) 50:2384-7. doi: 10.1007/s00125-007-0808-8

78. Bjornstad P, Maahs DM, Wadwa RP, Pyle L, Rewers M, Eckel RH, et al. Plasma triglycerides predict incident albuminuria and progression of coronary artery calcification in adults with type 1 diabetes: the coronary artery calcification in type 1 diabetes study. J Clin Lipidol. (2014) 8:57683. doi: $10.1016 /$ j.jacl. 2014.08 .008

79. Joannides R, Haefeli WE, Linder L, Richard V, Bakkali EH, Thuillez $\mathrm{C}$, et al. Nitric oxide is responsible for flow-dependent dilatation of human peripheral conduit arteries in vivo. Circulation. (1995) 91:13149. doi: 10.1161/01.CIR.91.5.1314

80. Shimokawa H, Yasutake H, Fujii K, Owada MK, Nakaike R, Fukumoto $\mathrm{Y}$, et al. The importance of the hyperpolarizing mechanism increases as the vessel size decreases in endothelium-dependent relaxations in rat mesenteric circulation. J Cardiovasc Pharmacol. (1996) 28:70311. doi: 10.1097/00005344-199611000-00014

81. Malakul W, Thirawarapan S, Suvitayavat W, Woodman OL. Type 1 diabetes and hypercholesterolaemia reveal the contribution of endothelium-derived hyperpolarizing factor to endothelium-dependent relaxation of the rat aorta. Clin Exp Pharmacol Physiol. (2008) 35:192-200. doi: 10.1111/j.1440-1681.2007.04811.x

82. Meeking DR, Browne DL, Allard S, Munday J, Chowienczyck PJ, Shaw KM, et al. Effects of cyclo-oxygenase inhibition on vasodilatory response to acetylcholine in patients with type 1 diabetes and nondiabetic subjects. Diabetes Care. (2000) 23:1840-3. doi: 10.2337/diacare.23. 12.1840

83. Chawla A, Chawla R, Jaggi S. Microvasular and macrovascular complications in diabetes mellitus: Distinct or continuum? Indian J Endocrinol Metab. (2016) 20:546-51. doi: 10.4103/2230-8210.183480

84. Makimattila S, Virkamaki A, Groop PH, Cockcroft J, Utriainen T, Fagerudd J, et al. Chronic hyperglycemia impairs endothelial function and insulin sensitivity via different mechanisms in insulin-dependent diabetes mellitus. Circulation. (1996) 94:1276-82. doi: 10.1161/01.CIR.94. 6.1276

85. Shah AS, Gao Z, Dolan LM, Dabelea D, D'Agostino RB Jr, Urbina EM. Assessing endothelial dysfunction in adolescents and young adults with type 1 diabetes mellitus using a non-invasive heat stimulus. Pediatr Diabetes. (2015) 16:434-40. doi: 10.1111/pedi.12189

86. Hoffman RP, Dye AS, Huang H, Bauer JA. Effects of glucose control and variability on endothelial function and repair in adolescents with type 1 diabetes. ISRN Endocrinol. (2013) 2013:876547. doi: 10.1155/2013/ 876547

87. Joshua IG, Zhang Q, Falcone JC, Bratcher AP, Rodriguez WE, Tyagi SC. Mechanisms of endothelial dysfunction with development of type 1 diabetes mellitus: role of insulin and C-peptide. J Cell Biochem. (2005) 96:114956. doi: $10.1002 /$ jcb. 20620

88. Milsom AB, Jones CJ, Goodfellow J, Frenneaux MP, Peters JR, James PE. Abnormal metabolic fate of nitric oxide in Type I diabetes mellitus. Diabetologia. (2002) 45:1515-22. doi: 10.1007/s00125-002-0956-9

89. Volianitis S, Secher NH. Cardiovascular control during whole body exercise. J Appl Physiol (1985). (2016) 121:37690. doi: 10.1152/japplphysiol.00674.2015

90. Barrett EJ, Eggleston EM, Inyard AC, Wang H, Li G, Chai W, et al. The vascular actions of insulin control its delivery to muscle and regulate the rate-limiting step in skeletal muscle insulin action. Diabetologia. (2009) 52:752-64. doi: 10.1007/s00125-009-1313-z

91. Dye AS, Huang H, Bauer JA, Hoffman RP. Hyperglycemia increases muscle blood flow and alters endothelial function in adolescents with type 1 diabetes. Exp Diabetes Res. (2012) 2012:170380. doi: 10.1155/2012/170380

92. Hirsch IB, Brownlee M. Should minimal blood glucose variability become the gold standard of glycemic control? J Diabetes Complications. (2005) 19:178-81. doi: 10.1016/j.jdiacomp.2004.10.001

93. Ceriello A, Esposito K, Piconi L, Ihnat MA, Thorpe JE, Testa R, et al. Oscillating glucose is more deleterious to endothelial function and oxidative stress than mean glucose in normal and type 2 diabetic patients. Diabetes. (2008) 57:1349-54. doi: 10.2337/db08-0063

94. Costantino S, Paneni F, Battista R, Castello L, Capretti G, Chiandotto S, et al. Impact of glycemic variability on chromatin remodeling, oxidative stress, and endothelial dysfunction in patients with type 2 diabetes and with target HbAlc levels. Diabetes. (2017) 66:2472-82. doi: 10.2337/ db17-0294

95. Farabi SS, Quinn L, Phillips S, Mihailescu D, Park C, Ali M, et al. Endothelial dysfunction is related to glycemic variability and quality and duration of sleep in adults with type 1 diabetes. J Cardiovasc Nurs. (2018) 33:E21E5. doi: 10.1097/JCN.0000000000000485

96. Gimenez M, Gilabert R, Monteagudo J, Alonso A, Casamitjana R, Pare C, et al. Repeated episodes of hypoglycemia as a potential aggravating factor for preclinical atherosclerosis in subjects with type 1 diabetes. Diabetes Care. (2011) 34:198-203. doi: 10.2337/dc10-1371

97. Joy NG, Perkins JM, Mikeladze M, Younk L, Tate DB, Davis SN. Comparative effects of acute hypoglycemia and hyperglycemia on pro-atherothrombotic biomarkers and endothelial function in non-diabetic humans. $J$ Diabetes Complications. (2016) 30:1275-81. doi: 10.1016/j.jdiacomp.2016. 06.030

98. Maiorino MI, Casciano O, Della Volpe E, Bellastella G, Giugliano D, Esposito K. Reducing glucose variability with continuous subcutaneous insulin infusion increases endothelial progenitor cells in type 1 diabetes: an observational study. Endocrine. (2016) 52:244-52. doi: 10.1007/s12020-015-0686-7

99. Desouza C, Salazar H, Cheong B, Murgo J, Fonseca V. Association of hypoglycemia and cardiac ischemia: a study based on continuous monitoring. Diabetes Care. (2003) 26:1485-9. doi: 10.2337/diacare.26.5.1485

100. Ghiadoni L, Faita F, Salvetti M, Cordiano C, Biggi A, Puato $\mathrm{M}$, et al. Assessment of flow-mediated dilation reproducibility: a nationwide multicenter study. J Hypertens. (2012) 30:1399405. doi: 10.1097/HJH.0b013e328353f222

101. Greyling A, van Mil AC, Zock PL, Green DJ, Ghiadoni L, Thijssen $\mathrm{DH}$, et al. Adherence to guidelines strongly improves reproducibility of brachial artery flow-mediated dilation. Atherosclerosis. (2016) 248:196202. doi: 10.1016/j.atherosclerosis.2016.03.011

102. Corretti MC, Anderson TJ, Benjamin EJ, Celermajer D, Charbonneau F, Creager MA, et al. Guidelines for the ultrasound assessment of endothelialdependent flow-mediated vasodilation of the brachial artery: a report of the International Brachial Artery Reactivity Task Force. J Am Coll Cardiol. (2002) 39:257-65. doi: 10.1016/S0735-1097(01)01746-6

103. Lockhart CJ, McVeigh GE, Cohn JN. Measuring endothelial function. Curr Diabetes Rep. (2006) 6:267-73. doi: 10.1007/s11892-006-0059-y

104. Mitchell GF, Parise H, Vita JA, Larson MG, Warner E, Keaney JF, et al. Local shear stress and brachial artery flow-mediated dilation: the Framingham Heart Study. Hypertension. (2004) 44:134-9. doi: 10.1161/01.HYP.0000137305.77635.68

105. Loader J, Roustit M, Taylor F, MacIsaac RJ, Stewart S, Lorenzen $\mathrm{C}$, et al. Assessing cutaneous microvascular function with iontophoresis: avoiding non-specific vasodilation. Microvasc Res. (2017) 113:29-39. doi: 10.1016/j.mvr.2017.04.006

106. Tagougui S, Fontaine P, Leclair E, Aucouturier J, Matran R, Oussaidene $\mathrm{K}$, et al. Regional cerebral hemodynamic response to incremental exercise is blunted in poorly controlled patients with uncomplicated type 1 diabetes. Diabetes Care. (2015) 38:858-67. doi: 10.2337/ dc14-1792

107. Musen G, Tinsley LJ, Marcinkowski KA, Pober D, Sun JK, Khatri $\mathrm{M}$, et al. Cognitive function deficits associated with long-duration type 
1 diabetes and vascular complications. Diabetes Care. (2018) 41:174956. doi: $10.2337 / \mathrm{dc} 17-1955$

108. Baylie RL, Brayden JE. TRPV channels and vascular function. Acta Physiol (Oxf). (2011) 203:99-116. doi: 10.1111/j.1748-1716.2010.02217.x

109. Huang A, Sun D, Jacobson A, Carroll MA, Falck JR, Kaley G. Epoxyeicosatrienoic acids are released to mediate shear stress-dependent hyperpolarization of arteriolar smooth muscle. Circ Res. (2005) 96:37683. doi: 10.1161/01.RES.0000155332.17783.26

110. Kelm M, Preik-Steinhoff H, Preik M, Strauer BE. Serum nitrite sensitively reflects endothelial NO formation in human forearm vasculature: evidence for biochemical assessment of the endothelial L-arginine-NO pathway. Cardiovasc Res. (1999) 41:765-72. doi: 10.1016/S0008-6363(98) 00259-4

111. Fuchsjager-Mayrl G, Pleiner J, Wiesinger GF, Sieder AE, Quittan M, Nuhr MJ, et al. Exercise training improves vascular endothelial function in patients with type 1 diabetes. Diabetes Care. (2002) 25:1795-801. doi: 10.2337/diacare.25. 10.1795
112. Ceriello A, Novials A, Ortega E, Canivell S, La Sala L, Pujadas G, et al. Vitamin C further improves the protective effect of glucagon-like peptide1 on acute hypoglycemia-induced oxidative stress, inflammation, and endothelial dysfunction in type 1 diabetes. Diabetes Care. (2013) 36:41048. doi: $10.2337 / \mathrm{dc} 13-0750$

Conflict of Interest: The authors declare that the research was conducted in the absence of any commercial or financial relationships that could be construed as a potential conflict of interest.

Copyright (C) 2020 Lespagnol, Dauchet, Pawlak-Chaouch, Balestra, Berthoin, Feelisch, Roustit, Boissière, Fontaine and Heyman. This is an open-access article distributed under the terms of the Creative Commons Attribution License (CC BY). The use, distribution or reproduction in other forums is permitted, provided the original author(s) and the copyright owner(s) are credited and that the original publication in this journal is cited, in accordance with accepted academic practice. No use, distribution or reproduction is permitted which does not comply with these terms. 